\title{
Uniform modular lattices and affine buildings
}

\author{
Hiroshi HIRAI \\ Department of Mathematical Informatics, \\ Graduate School of Information Science and Technology, \\ The University of Tokyo, Tokyo, 113-8656, Japan. \\ hirai@mist.i.u-tokyo.ac.jp
}

September 20, 2019

\begin{abstract}
In this paper, we present a simple lattice-theoretic characterization for affine buildings of type A. We introduce a class of modular lattices, called uniform modular lattices, and show that uniform modular lattices and affine buildings of type A constitute the same object. This is an affine counterpart of the well-known equivalence between projective geometries $(\simeq$ complemented modular lattices) and spherical buildings of type A.
\end{abstract}

Keywords: Modular lattice, affine building of type A

\section{Introduction}

Buildings, due to Tits [18] and Bruhat and Tits [4], are simplicial complexes that extract combinatorial properties of algebraic groups, and have numerous important applications in branches of mathematics; see [3, 17]. The present article addresses lattice-theoretic aspects of buildings. As is well-known, spherical buildings of type A and (generalized) projective geometries are the same mathematical object [18]: All chains (flags) of subspaces in a projective geometry form a spherical building of type A, and any spherical building of type A is obtained in this way. In lattice theory, a classical result [5] by Birkhoff says that the subspace lattice of a projective geometry is exactly a complemented modular lattice of finite rank (also known as a modular geometric lattice) - a modular lattice in which the maximum element is the join of atoms. Thus we can say:

Theorem 1.1 ([18]; see e.g., [17, THEOREM 4.1.4]). Complemented modular lattices of finite rank and spherical buildings of type A constitute the same object.

The goal of this paper is to establish an analogue of this theorem for affine buildings of type A. We introduce an affine analogue of complemented modular lattices, named uniform modular lattices. This class of modular lattices is simply defined: A modular lattice $\mathcal{L}$ is called uniform if the operator $x \mapsto$ (the join of all elements covering $x$ ) is an automorphism on $\mathcal{L}$. Our main result, which might be a reasonable affine counterpart of Theorem 1.1, is as follows. 
Theorem 1.2. Uniform modular lattices and affine buildings of type A constitute the same object.

The precise meaning of this theorem is explained in Theorems 3.6 and 3.16 . The former says that any uniform modular lattice $\mathcal{L}$ gives rise to an affine building of type $\mathrm{A}$ as the projection $\mathcal{C}(\mathcal{L})$ of a subcomplex of its order complex, and the latter constructs a uniform modular lattice $\mathcal{L}(\Delta)$ from any affine building $\Delta$ of type $\mathrm{A}$ for which $\mathcal{C}(\mathcal{L}(\Delta))=\Delta$.

By relaxing modularity to semimodularity, we obtain, in the same way, a further natural class of lattices, called uniform semimodular lattices. This class of lattices is studied in the companion paper [13].

After submitting this paper, we found a closely related approach by Abels [1]. He introduced the notion of semimodular lattices with cofinal $Z$-action, and studied, in a lattice-theoretic way, the gallery distance in the affine building of $\mathrm{SL}\left(K^{n}\right)$ for a field $K$ with a discrete valuation. He noticed that the affine building of $\mathrm{SL}\left(K^{n}\right)$ gives rise to a modular lattice with cofinal $Z$-action and that the simplicial structure of the building is described by this lattice. A uniform (semi)modular lattice $\mathcal{L}$ is indeed an example of (semi)modular lattices with cofinal $Z$-action, where the constructions of lattice $\mathcal{L}$ from the affine building of $\operatorname{SL}\left(K^{n}\right)$ and the simplicial complex $\mathcal{C}(\mathcal{L})$ from $\mathcal{L}$ coincide with that in [1]; see Example 3.3. Thus one can also say that the result in this paper pushes the lattice-theoretic approach of [1] toward general affine buildings of type A.

The rest of this paper is organized as follows. In Section 2, we introduce basic terminologies and results on lattices and buildings. In Section 3 , we formally introduce the concept of uniform modular lattices, and establish the main theorem (Theorem 1.2) by proving Theorems 3.6 and 3.16. In Section 4, we give some remarks that include future applications of uniform modular lattices to combinatorial optimization.

\section{Preliminaries}

Our references for lattices are [2, Chapter II], the first edition of [5], and [9]. The references for buildings are [3, 8, , 17].

\subsection{Basic notation}

Let $\mathbf{Z}$ and $\mathbf{R}$ be the set of integers and reals, respectively. Let $\mathbf{R}_{+}$denote the set of nonnegative reals. In $\mathbf{R}^{n}$, let $e_{i}$ denote the $i$ th unit vector, $\mathbf{0}$ the zero vector, and $\mathbf{1}$ the all-one vector. For $x, y \in \mathbf{R}^{n}$, by $x \leq y$ we mean that $x_{i} \leq y_{i}$ for all $i=1,2, \ldots, n$. Let $\min (x, y)$ and $\max (x, y)$ be defined as the vectors in $\mathbf{R}^{n}$ obtained from $x, y$ by taking minimum and maximum componentwise, respectively.

Lattices. We will use the basic terminology of poset and lattice. A poset (partially ordered set) $\mathcal{L}$ is a set endowed with a partial order relation $\preceq$, where $x \prec y$ is meant as $x \preceq y$ and $x \neq y$. The opposite $\check{\mathcal{L}}$ of $\mathcal{L}$ is the poset on $\mathcal{L}$ with partial order $\preceq^{\prime}$ defined by $x \preceq \preceq^{\prime} y \Leftrightarrow y \preceq x$. The partial order of the direct product $\mathcal{L} \times \mathcal{L}^{\prime}$ of two posets $\mathcal{L}, \mathcal{L}^{\prime}$ is defined as $\left(x, x^{\prime}\right) \preceq\left(y, y^{\prime}\right) \Leftrightarrow x \preceq y$ and $x^{\prime} \preceq y^{\prime}$.

For elements $x, y$ with $x \preceq y$, the interval of $x, y$ is the set of elements $z$ with $x \preceq z \preceq y$, and is denoted by $[x, y]$. We say that $y$ covers $x$ if $x \neq y$ and $[x, y]=\{x, y\}$. 
A totally ordered subset $\mathcal{C}$ of $\mathcal{L}$ is called a chain. If a chain $\mathcal{C}$ consists of $x_{0}, x_{1}, \ldots, x_{m}, \ldots$ with $x_{i} \prec x_{i+1}$ for all indices $i$, then $\mathcal{C}$ is denoted by $\left(x_{0} \prec x_{1} \prec \cdots \prec x_{m} \prec \cdots\right)$. The length of chain $\mathcal{C}$ is defined as its cardinality $|\mathcal{C}|$ minus one. The unique minimal common upper bound of elements $x, y$ is called the join of $x, y$, and is denoted by $x \vee y$ if it exists. The unique maximal common lower bound of $x, y$ is called the meet of $x, y$, and is denoted by $x \wedge y$ if it exists. A lattice $\mathcal{L}$ is a poset such that every pair of elements has the join and meet. An isomorphism between two lattices $\mathcal{L}$ and $\mathcal{L}^{\prime}$ is a bijection $\varphi: \mathcal{L} \rightarrow \mathcal{L}^{\prime}$ such that $\varphi(x \wedge y)=\varphi(x) \wedge \varphi(y)$ and $\varphi(x \vee y)=\varphi(x) \vee \varphi(y)$ for all $x, y \in \mathcal{L}$, or equivalently, $\varphi(x) \preceq \varphi(y) \Leftrightarrow x \preceq y$ for all $x, y \in \mathcal{L}$. In addition, if $\mathcal{L}=\mathcal{L}^{\prime}$, then $\varphi$ is called an automorphism on $\mathcal{L}$. For a subset $S \subseteq \mathcal{L}$, the unique maximal lower bound of $S$ (the meet of $S$ ) is denoted by $\bigwedge S$ if it exists, and the unique minimal upper bound of $S$ (the join of $S$ ) is denoted by $\bigvee S$ if it exists. In a lattice $\mathcal{L}$, the minimum element $\bigwedge \mathcal{L}$, if it exists, is denoted by $\overline{0}$, and the maximum element $\bigvee \mathcal{L}$, if it exists, is denoted by $\overline{1}$. In a lattice $\mathcal{L}$ having the minimum element $\overline{0}$, the rank $r(x)$ of element $x$ is the maximum length of a chain in $[\overline{0}, x]$. The rank of $\mathcal{L}$ (having $\overline{0}$ and $\overline{1}$ ) is the maximum length of a maximal chain of $\mathcal{L}$. By an atom we mean an element of rank one. A sublattice of a lattice $\mathcal{L}$ is a subset $\mathcal{L}^{\prime} \subseteq \mathcal{L}$ with the property that $x, y \in \mathcal{L}^{\prime}$ imply $x \wedge y, x \vee y \in \mathcal{L}^{\prime}$. Intervals are sublattices. In this paper, any lattice satisfies the following finiteness assumption:

(F) Any interval $[x, y]$ has a finite $\operatorname{rank} r[x, y]<\infty$.

Simplicial complexes. A simplicial complex $\Delta$ is a family of subsets of a nonempty set $V$ such that $A^{\prime} \subseteq A \in \Delta$ implies $A^{\prime} \in \Delta$. A member $A$ in $\Delta$ is called a simplex, and its dimension is defined as $|A|-1$. The dimension of $\Delta$ is defined as the maximum dimension of a simplex in $\Delta$. A 0 -dimensional simplex is called a vertex. The set of vertices is denoted by $\Delta^{0}$. We assume that all singleton $\{v\}(v \in V)$ are vertices, and hence $\Delta^{0}$ is identified with $V$. Two simplicial complexes $\Delta, \Delta^{\prime}$ are isomorphic if there exists a bijection $\rho: \Delta^{0} \rightarrow \Delta^{\prime 0}$, called an isomorphism, such that $A \in \Delta \Leftrightarrow \rho(A) \in \Delta^{\prime}$. An isomorphism $\rho$ induces an inclusion-preserving bijection $\Delta \rightarrow \Delta^{\prime}$ by $A \mapsto \rho(A)$; therefore $\rho$ is also regarded as $\Sigma \rightarrow \Sigma^{\prime}$.

For a poset $\mathcal{P}$, the order complex $\mathcal{O}(\mathcal{P})$ of $\mathcal{P}$ is the simplicial complex on $\mathcal{P}$ consisting of all chains of finite length.

A geometric realization $|\Delta|$ of $\Delta$ is the set of all functions $u: \Delta^{0} \rightarrow \mathbf{R}_{+}$such that $\left\{x \in \Delta^{0} \mid u(x)>0\right\} \in \Delta$ and $\sum_{x \in \Delta^{0}} u(x)=1$. Then, abstract simplices in $\Delta$ become geometric simplices in $|\Delta|$ with mutually disjoint relative interiors.

\section{$2.2 \quad$ Modular lattices}

A lattice $\mathcal{L}$ is called modular if $(y \wedge z) \vee x=(x \vee z) \wedge y$ for all triples $x, y, z \in \mathcal{L}$ with $x \preceq y$. Modular lattices satisfy the Jordan-Dedekind chain condition:

(JD) Maximal chains in any interval have the same length.

A valuation of a lattice $\mathcal{L}$ is a function $v: \mathcal{L} \rightarrow \mathbf{R}$ satisfying $v(x)<v(y)$ for all $x, y \in \mathcal{L}$ with $x \prec y$, and

$$
v(x)+v(y)=v(x \wedge y)+v(x \vee y) \quad(x, y \in \mathcal{L}) .
$$


It is well-known that the rank function of a modular lattice having $\overline{0}$ is a valuation; see e.g., [2, Theorem 2.27]. Conversely the existence of a valuation implies the modularity.

Lemma 2.1 (see [5, Theorem 3.11]). A lattice $\mathcal{L}$ having a valuation is a modular lattice.

Sketch of proof. For $x, y, z \in \mathcal{L}$ with $x \preceq y$, it always holds $(y \wedge z) \vee x \preceq(x \vee z) \wedge y$. If $\mathcal{L}$ has a valuation $v$, by using (2.1), one can see that $v((y \wedge z) \vee x)=v((x \vee z) \wedge y)$, which implies $(y \wedge z) \vee x=(x \vee z) \wedge y$.

This proof uses the following obvious rank-comparison argument, which will be often used later:

(rc) $x \preceq y$ and $v(x)=v(y)$ imply $x=y$.

For a subset $S$ of lattice $\mathcal{L}$, let $\langle S\rangle$ denote the sublattice of $\mathcal{L}$ generated by $S$ (= the minimum sublattice containing $S$ ).

Lemma 2.2 (See [9, Theorems 348, 364]). Let $\mathcal{L}$ be a modular lattice. For $p, q \in \mathcal{L}$, the following hold:

(1) $[p, p \vee q]$ is isomorphic to $[p \wedge q, q]$ by $x \mapsto x \wedge q$ with inverse $y \mapsto y \vee p$.

(2) $\langle[p \wedge q, p] \cup[p \wedge q, q]\rangle$ is isomorphic to $[p \wedge q, p] \times[p \wedge q, q]$ by $x \mapsto(x \wedge p, x \wedge q)$ with inverse $(u, v) \mapsto u \vee v$.

In a lattice $\mathcal{L}$ with $\overline{0}$ and $\overline{1}$, a complement of an element $x \in \mathcal{L}$ is an element $y$ such that $x \wedge y=\overline{0}$ and $x \vee y=\overline{1}$. A lattice $\mathcal{L}$ with $\overline{0}$ and $\overline{1}$ is said to be complemented if every element has a complement. The following facts are basic; see e.g., [5, Theorem 4.1].

(cm1) A modular lattice is complemented if and only if $\overline{1}$ is the join of atoms.

(cm2) Every interval of a complemented modular lattice is complemented modular.

(cm3) The opposite of a complemented modular lattice is complemented modular.

Note that $(\mathrm{cm} 3)$ is immediate from the definition, and (cm1) is true under the assumption (F); in particular, modular geometric lattices (see [2, Section II. 3]) and complemented modular lattices are the same in this paper.

In a complemented modular lattice $\mathcal{L}$ of rank $n$, a set of $k$ atoms $a_{1}, a_{2}, \ldots, a_{k}$ is said to be independent if $r\left(a_{1} \vee a_{2} \vee \cdots \vee a_{k}\right)=k$, or equivalently if $a_{i} \wedge \bigvee_{j \neq i} a_{j}=\overline{0}$ for all $i$. A basis of $\mathcal{L}$ is a set of $n$ independent atoms.

Example 2.3. The partial order $\preceq$ on $\{0,1\}^{n}$ is defined as the vector order $\leq$. Then $\{0,1\}^{n}$ is a complemented modular lattice of rank $n$ (more precisely it is a Boolean lattice). The meet and join are given by $u \wedge v=\min (u, v)$ and $u \vee v=\max (u, v)$. Unit vectors $e_{1}, e_{2}, \ldots, e_{n}$ form the unique basis of $\{0,1\}^{n}$.

Example 2.4. Let $\mathcal{L}$ be the family of all vector subspaces $X$ of a vector space $V$ of dimension $n$. Regard $\mathcal{L}$ as a poset with respect to inclusion order $\subseteq$. Then $\mathcal{L}$ is a complemented modular lattice of rank $n$, where $\wedge$ and $\vee$ are equal to $\cap$ and + , respectively, and $X \mapsto \operatorname{dim} X$ is a valuation (and the rank function). A basis of $\mathcal{L}$ is precisely the set of 1-dimensional vector spaces corresponding to a basis of $V$. 
In the following three lemmas, $\mathcal{L}$ is assumed to be a complemented modular lattice of rank $n$.

Lemma 2.5. Let $C$ be a maximal chain in $\mathcal{L}$, and $p$ an element in $\mathcal{L}$.

(1) There exists a complement $q$ of $p$ in $\mathcal{L}$ such that the sublattice $\langle[\overline{0}, p] \cup[\overline{0}, q]\rangle$ contains $C$.

(2) In addition, if a basis $a_{1}, a_{2}, \ldots, a_{k}$ of $[\overline{0}, p]$ generates $C \wedge p$ and $a$ basis $b_{1}, b_{2}, \ldots, b_{l}$ of $[p, \overline{1}]$ generates $C \vee p$, then $a_{1}, a_{2}, \ldots, a_{k}, b_{1} \wedge q, b_{2} \wedge q, \ldots, b_{l} \wedge q$ is a basis of $\mathcal{L}$ that generates $C$.

Proof. (1). We use induction on the rank $n$ of $\mathcal{L}$. Suppose $C=\left(\overline{0}=x_{0} \prec x_{1} \prec \cdots \prec\right.$ $\left.x_{n}=\overline{1}\right)$. We may assume that $n \geq 2$.

Case 1: $p \preceq x_{n-1}$. Consider interval $\left[\overline{0}, x_{n-1}\right]$, which is complemented modular by (cm2), and consider maximal chain $C^{\prime}=\left(\overline{0}=x_{0} \prec x_{1} \prec \cdots \prec x_{n-1}\right)$ in $\left[\overline{0}, x_{n-1}\right]$. By induction, there is a complement $q^{\prime} \in\left[\overline{0}, x_{n-1}\right]$ of $p$ such that $\left\langle[\overline{0}, p] \cup\left[\overline{0}, q^{\prime}\right]\right\rangle$ contains $C^{\prime}$. Now $q^{\prime} \preceq x_{n-1} \prec x_{n}=\overline{1}$. We can choose a complement $q \in\left[q^{\prime}, \overline{1}\right]$ of $x_{n-1}$ (which covers $\left.q^{\prime}\right)$. Then $q$ is a desired complement of $p$ in $\mathcal{L}$. Indeed, $p \vee q=p \vee q^{\prime} \vee q=x_{n-1} \vee q=\overline{1}$. Also $p \wedge q=\overline{0}$ follows from (rc) and $r(q)-r(\overline{0})=1+r\left(q^{\prime}\right)-r(\overline{0})=1+r\left(x_{n-1}\right)-r(p)=$ $r(\overline{1})-r(p)=r(q)-r(p \wedge q)$ (implying $r(\overline{0})=r(p \wedge q))$.

Case 2: $p \npreceq x_{n-1}$. Consider $p^{\prime}:=x_{n-1} \wedge p$. Then $p^{\prime}$ is covered by $p$; consider (2.1) for the setting $v=r, x=x_{n-1}$, and $y=p$. As above, consider complemented modular lattice $\left[\overline{0}, x_{n-1}\right]$ and maximal chain $C^{\prime}$. By induction, there is a complement $q \in\left[\overline{0}, x_{n-1}\right]$ of $p^{\prime}$ such that $\left\langle\left[\overline{0}, p^{\prime}\right] \cup[\overline{0}, q]\right\rangle$ contains $C^{\prime}$. Since $p \vee q=\overline{1}$, the sublattice $\langle[\overline{0}, p] \cup[\overline{0}, q]\rangle$ contains $C$.

(2). Consider $x \in C$. Then $x \wedge p$ is the join of a subset of $a_{1}, a_{2}, \ldots, a_{k}$, say $x \wedge p=a_{1} \vee a_{2} \vee \cdots \vee a_{k^{\prime}}$. Also $x \vee p$ is represented as $x \vee p=b_{1} \vee b_{2} \vee \cdots \vee b_{l^{\prime}}$. Now $x=(x \wedge p) \vee(x \wedge q)=(x \vee p) \wedge(x \vee q)$ holds by Lemma 2.2 (2) (applied to the opposite). From $x=(x \vee p) \wedge(x \vee q)$, we have $x \wedge q=(x \vee p) \wedge(x \vee q) \wedge q=(x \vee p) \wedge q$. Thus $x=(x \wedge p) \vee((x \vee p) \wedge q)=a_{1} \vee a_{2} \vee \cdots \vee a_{k^{\prime}} \vee\left(\left(b_{1} \vee b_{2} \vee \cdots \vee b_{l^{\prime}}\right) \wedge q\right)=$ $a_{1} \vee a_{2} \vee \cdots \vee a_{k^{\prime}} \vee\left(b_{1} \wedge q\right) \vee\left(b_{2} \wedge q\right) \vee \cdots \vee\left(b_{l^{\prime}} \vee q\right)$, where the last equality follows from Lemma 2.2 (1) and $b_{i} \succeq p$. Therefore $a_{1}, a_{2}, \ldots, a_{k}, b_{1} \wedge q, b_{2} \wedge q, \ldots, b_{l} \wedge q$ generate $C$. They form a basis since their join equals $\overline{1}$ (by Lemma 2.2) with $k+l=n$.

The sublattice $\left\langle a_{1}, a_{2}, \ldots, a_{n}\right\rangle$ generated by a basis $a_{1}, a_{2}, \ldots, a_{n}$ is isomorphic to Boolean lattice $\{0,1\}^{n}$ by $e_{i_{1}}+e_{i_{2}}+\cdots+e_{i_{k}} \mapsto a_{i_{1}} \vee a_{i_{2}} \vee \cdots \vee a_{i_{k}}$. We call such a sublattice a $\{0,1\}^{n}$-skeleton. The next lemma is a folklore in theory of modular lattice, and is essentially one of axioms of building; see (B2) in Section 2.3 .

Lemma 2.6 (See [9, Theorem 363]). For two chains $C, D$ in $\mathcal{L}$, there exists a $\{0,1\}^{n}$ skeleton in $\mathcal{L}$ containing $C$ and $D$.

Proof. We use induction on $n$; the case of $n=1$ is obvious. Thus $n \geq 2$. We may assume that $C=\left(\overline{0}=x_{0} \prec x_{1} \prec \cdots \prec x_{n}=\overline{1}\right)$ and $D=\left(\overline{0}=y_{0} \prec y_{1} \prec \cdots \prec y_{n}=\overline{1}\right)$. Consider complemented modular lattice $\left[\overline{0}, x_{n-1}\right]$, and maximal chains $C^{\prime}:=\left(\overline{0}=x_{0} \prec\right.$ $\left.x_{1} \prec \cdots \prec x_{n-1}\right)$ and $D^{\prime}:=\left(\overline{0}=y_{0} \wedge x_{n-1} \preceq y_{1} \wedge x_{n-1} \preceq \cdots \preceq y_{n} \wedge x_{n-1}=x_{n-1}\right)$. By induction, there is a $\{0,1\}^{n-1}$-skeleton $\mathcal{F}^{\prime}=\left\langle a_{1}, a_{2}, \ldots, a_{n-1}\right\rangle$ in $\left[\overline{0}, x_{n-1}\right]$ containing $C^{\prime}, D^{\prime}$. By the previous lemma, we can choose a complement $a$ of $x_{n-1}$ in $\mathcal{L}$ such that $\left\langle\left[\overline{0}, x_{n-1}\right] \cup[\overline{0}, a]\right\rangle$ contains $D$. Here $a$ is an atom of $\mathcal{L}$, and $a_{1}, a_{2}, \ldots, a_{n-1}, a$ form a 
basis of $\mathcal{L}$. Thus the $\{0,1\}^{n}$-skeleton $\mathcal{F}:=\left\langle a_{1}, a_{2}, \ldots, a_{n-1}, a\right\rangle$ contains $C$ and $D$, as required.

Let $C=\left(\overline{0}=x_{0} \prec x_{1} \prec \cdots \prec x_{n}=\overline{1}\right)$ be a maximal chain in $\mathcal{L}$. The relative position $y_{C} \in\{0,1\}^{n}$ of $y \in \mathcal{L}$ with respect to $C$ is defined as follows. Choose a $\{0,1\}^{n}$ skeleton $\mathcal{F}$ containing $C$, y via Lemma 2.6. Regard $\mathcal{F}$ as $\{0,1\}^{n}$, where we assume $x_{i}=x_{i-1}+e_{i}$ for $i=1,2, \ldots, n$ by relabeling. Define the relative position $y_{C} \in\{0,1\}^{n}$ as the 0,1 -vector corresponding to $y$ in this coordinate $\mathcal{F}$.

Lemma 2.7. The relative position $y_{C}$ of $y$ is independent of the choice of $a\{0,1\}^{n}$ skeleton containing $C, y$.

Proof. $y_{C}$ is exactly the sum of unit vectors $e_{i}$ over indices $i$ with $e_{i} \leq y\left(=y_{C}\right)$. Here $e_{i} \leq y\left(\Leftrightarrow e_{i} \preceq y\right)$ is equivalent to the lattice condition $x_{i} \wedge y \succ x_{i-1} \wedge y(\Leftrightarrow$ $\left.\min \left(x_{i}, y\right)-\min \left(x_{i-1}, y\right)=e_{i}\right)$, which is independent of the $\{0,1\}^{n}$-skeleton.

\subsection{Spherical/affine buildings of type A}

We first introduce the spherical/affine Coxeter complex of type A (without grouptheoretic language). We consider the decomposition of $\mathbf{R}^{n} / \mathbf{R} \mathbf{1}$ (the quotient space of $\mathbf{R}^{n}$ by $\left.\mathbf{R} \mathbf{1}\right)$ by the following hyperplanes in $\mathbf{R}^{n}$ :

$$
H_{i j}:=\left\{x \in \mathbf{R}^{n} \mid x_{i}=x_{j}\right\} \quad(1 \leq i<j \leq n) .
$$

The closure of each connected component of $\left(\mathbf{R}^{n} \backslash \bigcup_{1 \leq i<j \leq n} H_{i j}\right) / \mathbf{R} 1$ is a simplicial cone that is the conical hull of $n-1$ vectors

$$
e_{\sigma(1)}, e_{\sigma(1)}+e_{\sigma(2)}, \ldots, e_{\sigma(1)}+e_{\sigma(2)}+\cdots+e_{\sigma(n-1)}
$$

modulo R1 for a permutation $\sigma$ on $\{1,2, \ldots, n\}$. The spherical Coxeter complex of type $A$ is the simplicial complex on $\{0,1\}^{n} \backslash\{\mathbf{0}, \mathbf{1}\}$ whose maximal simplices have vertices of form (2.3).

Next we introduce the affine Coxeter complex of type A. Consider the decomposition of $\mathbf{R}^{n} / \mathbf{R} \mathbf{1}$ by the following affine hyperplanes:

$$
H_{i j, k}:=\left\{x \in \mathbf{R}^{n} \mid x_{i}=x_{j}+k\right\} \quad(1 \leq i<j \leq n, k \in \mathbf{Z}) .
$$

The closure of each connected component of $\left(\mathbf{R}^{n} \backslash \bigcup_{1 \leq i<j \leq n, k \in \mathbf{Z}} H_{i j, k}\right) / \mathbf{R} \mathbf{1}$ is a simplex that is the convex hull of $n$ vertices

$$
z, z+e_{\sigma(1)}, z+e_{\sigma(1)}+e_{\sigma(2)}, \ldots, z+e_{\sigma(1)}+e_{\sigma(2)}+\cdots+e_{\sigma(n-1)}
$$

modulo $\mathbf{R} 1$ for a permutation $\sigma$ on $\{1,2, \ldots, n\}$ and $z \in \mathbf{Z}^{n}$. The affine Coxeter complex of type $A$ is the simplicial complex on $\mathbf{Z}^{n} / \mathbf{R} \mathbf{1}$ whose maximal simplices have vertices of form $(2.5)$.

A spherical/affine building of type $A$ is a simplicial complex $\Delta$ having a family of subcomplexes, called apartments, satisfying the following axiom:

(B1) Each apartment is isomorphic to the spherical/affine Coxeter complex of type A.

(B2) For two simplices $A, B \in \Delta$, there is an apartment $\Sigma$ containing $A, B$. 
(B3) If two apartments $\Sigma, \Sigma^{\prime}$ contain simplices $A, B$, then there is an isomorphism $\varphi: \Sigma \rightarrow \Sigma^{\prime}$ fixing $A$ and $B$ pointwise, i.e., $\varphi(x)=x$ for $x \in A \cup B$.

The definition of general spherical/affine buildings is obtained by using general spherical/affine Coxeter complex in axiom (B1). The basic properties of buildings that we will use are summarized as follows; see [3, Chapter 4]. They hold for general spherical/affine buildings, not restricted to type A.

(col) A spherical/affine building (of type A) with dimension $n$ admits a labeling $\ell$ : $\Delta^{0} \rightarrow\{0,1,2, \ldots, n\}$, called a coloring, with the property that any distinct vertices $x, y$ in any simplex have distinct colors $\ell(x) \neq \ell(y)$. A coloring is automatically determined from any coloring (i.e., bijection to $\{0,1, \ldots, n\}$ ) of any maximal simplex, Moreover any two colorings $\ell, \ell^{\prime}$ are equivalent in the sense that $\ell^{\prime}=\kappa \circ \ell$ holds for some bijection $\kappa$ on $\{0,1,2, \ldots, n\}$.

$\left(\mathrm{B}^{+}\right)$The isomorphism $\varphi$ in (B3) can be taken to be color-preserving, i.e., $\ell(\varphi(x))=$ $\ell(x)$.

(ret) For an apartment $\Sigma$ and a maximal simplex $A$ in $\Sigma$, define a map $\rho_{\Sigma, A}: \Delta \rightarrow \Sigma$ as follows: For $B \in \Delta$, choose an apartment $\Sigma^{\prime}$ containing $A$ and $B$ via (B2), an isomorphism $\varphi: \Sigma^{\prime} \rightarrow \Sigma$ fixing $A$ via (B3), and let $\rho_{\Sigma, A}(B):=\varphi(B)$. This map $\rho_{\Sigma, A}$ is independent of the choice of an apartment $\Sigma^{\prime}$, and is a (color-preserving) retraction to $\Sigma$, i.e., $\rho_{\Sigma, A}(B)=B$ for $B \in \Sigma$. The map $\rho_{\Sigma, A}$ is called the canonical retraction.

The geometric realization $|\Delta|$ of an affine building $\Delta$ (of type A) admits a natural "Euclidean" metric; see [3, Chapter 11]. As seen above, the affine Coxeter complex $\Sigma$ is a triangulation of $\mathbf{R}^{n} / \mathbf{R} \mathbf{1}$, and the geometric realization $|\Sigma|$ is naturally regarded as $\mathbf{R}^{n} / \mathbf{R} \mathbf{1}$. Define a Euclidean metric $d_{\Sigma}$ on $|\Sigma|=\mathbf{R}^{n} / \mathbf{R} \mathbf{1}$ by $d_{\Sigma}(x+\mathbf{R} \mathbf{1}, y+\mathbf{R} \mathbf{1}):=$ $\|\bar{x}-\bar{y}\|_{2}$, where $\bar{x}$ is the unique vector with $\bar{x}-x \in \mathbf{R} \mathbf{1}$ and $\sum_{i=1}^{n} \bar{x}_{i}=0$. For two points $x, y$ in the geometric realization $|\Delta|$ of an affine building $\Delta$, define $d(x, y):=d_{\Sigma}(x, y)$ by choosing an apartment $\Sigma$ with $x, y \in|\Sigma|$ (via (B2)). In fact, $d(x, y)$ is independent of the choice of an apartment $\Sigma$. Hence $d$ is a well-defined distance function, and $|\Delta|$ becomes a metric space. The metric space $|\Delta|$ has a nice property on geodesics, where a geodesic between two point $x, y$ is a path $\gamma:[0,1] \rightarrow|\Delta|$ with $\gamma(0)=x, \gamma(1)=y$, and $d(\gamma(s), \gamma(t))=|\gamma(s)-\gamma(t)| d(x, y)$ for $s, t \in[0,1]$.

(geo) $|\Delta|$ is uniquely geodesic, that is, there is a unique geodesic between any two points $x, y \in|\Delta|$.

This property is a consequence of the fact that $|\Delta|$ is a $C A T(0)$ space; see [3, 6].

In the following, we explain the relationship (of Theorem 1.1) between complemented modular lattices and spherical buildings of type A. We here provide a larger part of the proof, since there seems no reference including such a direct proof without group-theory and incidence-geometry arguments, and the proof of Theorem 1.2 goes completely in parallel.

Notice that the spherical Coxeter complex of type A is nothing but the order complex of poset $\{0,1\}^{n} \backslash\{\mathbf{0}, \mathbf{1}\}$.

Theorem 2.8 ([18]). Let $\mathcal{L}$ be a complemented modular lattice of rank $n \geq 3$. Then the order complex $\mathcal{O}(\mathcal{L} \backslash\{\overline{0}, \overline{1}\})$ is a spherical building of type $A$ with dimension $n-2$. 
Proof. We show that subcomplexes of $\mathcal{O}(\mathcal{L})$ induced by $\{0,1\}^{n}$-skeletons (deleted by $\mathbf{0 , 1}$ ) satisfy the axiom of apartment. They are obviously isomorphic to the spherical Coxeter complex of type A, implying (B1). Consider two maximal simplices $A, B$, where $A \cup\{\overline{0}, \overline{1}\}$ and $B \cup\{\overline{0}, \overline{1}\}$ are maximal chains in $\mathcal{L}$. By Lemma 2.6, there is a $\{0,1\}^{n}$-skeleton containing $A, B$. This implies (B2). Suppose that two $\{0,1\}^{n}$-skeletons $\mathcal{F}, \mathcal{G}$ contain $A, B$. Suppose further that $\mathcal{F}=\left\langle f_{1}, f_{2}, \ldots, f_{n}\right\rangle$ and $\mathcal{G}=\left\langle g_{1}, g_{2}, \ldots, g_{n}\right\rangle$ for bases $f_{1}, f_{2}, \ldots, f_{n}$ and $g_{1}, g_{2}, \ldots, g_{n}$ of $\mathcal{L}$. By renumbering, we can assume that $A=\left\{g_{1} \vee g_{2} \vee \cdots \vee g_{k}\right\}_{k=1,2, \ldots, n-1}=\left\{f_{1} \vee f_{2} \vee \cdots \vee f_{k}\right\}_{k=1,2, \ldots, n-1}$. Define $\varphi: \mathcal{F} \rightarrow \mathcal{G}$ by $f_{i_{1}} \vee f_{i_{2}} \vee \cdots \vee f_{i_{k}} \mapsto g_{i_{1}} \vee g_{i_{2}} \vee \cdots \vee g_{i_{k}}$, which obviously induces an isomorphism between $\mathcal{O}(\mathcal{F})$ and $\mathcal{O}(\mathcal{G})$. Also $\varphi(x)=x$ for $x \in A$. For $x \in \mathcal{F} \cap \mathcal{G}$, if $x=f_{i_{1}} \vee f_{i_{2}} \vee \cdots \vee f_{i_{k}}$, i.e., $e_{i_{1}}+e_{i_{2}}+\cdots+e_{i_{k}}$ is the relative position of $x$ with respect to $A \cup\{\overline{0}, \overline{1}\}$, then $x=g_{i_{1}} \vee g_{i_{2}} \vee \cdots \vee g_{i_{k}}$ holds by Lemma 2.7, and hence $\varphi(x)=x$. Thus $\varphi$ is the identity on $\mathcal{F} \cap \mathcal{G}$, and consequently fixes $B$ pointwise, implying (B3).

Next we construct a complemented modular lattice from a spherical building $\Delta$ of type A. Our construction uses a special coloring; see (col) for colorings. A natural coloring is a coloring $\ell$ with the property that for every apartment $\Sigma$ there is an isomorphism $\rho: \Sigma \rightarrow \mathcal{O}\left(\{0,1\}^{n} \backslash\{\mathbf{0}, \mathbf{1}\}\right)$ with $\ell(x)=\sum_{i=1}^{n} \rho(x)_{i}$ for all $x \in \Sigma^{0}$. In fact, a natural coloring coincides with the natural ordering in the sense of [18]. In general, a coloring is obtained, in group-theoretic way, by associating each vertex with one of generators of the Coxeter group corresponding to the Coxeter complex. A natural ordering is the ordering of the generators so that consecutive numbers are assigned to adjacent generators in the Dynkin diagram of type A (that is a path).

Lemma 2.9. A natural coloring exists.

Sketch of proof. Consider an arbitrary apartment $\Sigma$. Then $\Sigma$ is isomorphic to the order complex $\mathcal{O}\left(\{0,1\}^{n} \backslash\{\mathbf{0}, \mathbf{1}\}\right)$. Identify $\Sigma^{0}$ with $\{0,1\}^{n} \backslash\{\mathbf{0}, \mathbf{1}\}$. Define the color $\ell(x)$ of $x \in \Sigma^{0}$ as $\sum_{i=1}^{n} x_{i}$. This is a coloring of $\Sigma$. Extend this coloring to the whole coloring $\ell$ on $\Sigma^{0}$ (via $\left.(\mathrm{col})\right)$. In fact, $\ell$ is a natural coloring. One can see this fact by counting and comparing the numbers of neighbors of a vertex with respect to their color. We will do this for the affine case in the proof of Lemma 3.15. The proof goes along precisely the same way.

Fix an arbitrary natural coloring $\ell$. Define a partial order $\preceq$ on $\Delta^{0}$ by $x \preceq y$ if $x$ and $y$ belong to a common simplex and $\ell(x) \leq \ell(y)$. It turns out in the next proposition that $\preceq$ is a partial order. Add a minimum element $\overline{0}$ and a maximal element $\overline{1}$ to $\Delta^{0}$. The resulting poset is denoted by $\mathcal{L}(\Delta)$.

Theorem 2.10 ([18]). Let $\Delta$ be a spherical building of type $A$. Then $\mathcal{L}(\Delta)$ is a complemented modular lattice, where $\Delta$ is equal to the order complex of $\mathcal{L}(\Delta) \backslash\{\overline{0}, \overline{1}\}$.

Proof. We first show that $\preceq$ is a partial order. It suffices to show that $x \preceq y$ and $y \preceq z$ imply $x \preceq z$. Consider an apartment $\Sigma$ containing simplices $\{x, y\}$ and $\{y, z\}$. Regard $\Sigma=\mathcal{O}\left(\{0,1\}^{n} \backslash\{\mathbf{0}, \mathbf{1}\}\right)$, where we can assume that $\ell(x)=\sum_{i} x_{i}$ since $\ell$ is natural. Then $x \leq y$ and $y \leq z$ in $\{0,1\}^{n}$. Hence $x \leq z$ holds in $\{0,1\}^{n}$. Consequently, $x$ and $z$ belong to a common simplex in $\Sigma \subseteq \Delta$, implying $x \preceq z$. In particular, $\Delta$ is the order complex of $\mathcal{L}(\Delta) \backslash\{\overline{0}, \overline{1}\}$.

Next we show that $\mathcal{L}(\Delta)$ is a lattice. Consider two vertices $x, y$. Suppose that $x$ and $y$ have two different maximal common lower bounds $z, z^{\prime}(\succ \overline{0})$. Consider an 
apartment $\Sigma$ containing $\{x, z\}$ and $\left\{y, z^{\prime}\right\}$. Regard $\Sigma=\mathcal{O}\left(\{0,1\}^{n} \backslash\{\mathbf{0}, \mathbf{1}\}\right)$. Then $z \leq \min (x, y) \geq z^{\prime}$, and $\min (x, y)$ is a common lower bound of $x$ and $y$. This contradicts the maximality of $z, z^{\prime}$. Thus the meet $x \wedge y$ exists, and is necessarily equal to $\min (x, y)$ in this apartment. Similarly the join $x \vee y$ exists, and is equal to $\max (x, y)$. In particular, we have $\ell(x)+\ell(y)=\sum_{i} x_{i}+\sum_{i} y_{i}=\sum_{i} \max (x, y)_{i}+\sum_{i} \min (x, y)_{i}=\ell(x \vee y)+\ell(x \wedge y)$, where we let $\ell(\overline{0}):=0$ and $\ell(\overline{1}):=n$. Thus $\ell$ is a valuation, and $\mathcal{L}(\Delta)$ is a modular lattice. Also $\overline{1}$ is the join of atoms (vertices having color 1 ). Indeed, $\overline{1}=\mathbf{1}=\sum_{i} e_{i}=$ $\bigvee_{i} e_{i}$ in any apartment.

\section{Uniform modular lattices}

In this section, we introduce the concept of uniform modular lattices (carefully) and establish the relation (Theorem 1.2) to affine buildings of type A. The ascending operator of a lattice $\mathcal{L}$ is a map $(\cdot)^{+}: \mathcal{L} \rightarrow \mathcal{L}$ defined by

$$
(x)^{+}:=\bigvee\{y \in \mathcal{L} \mid y \operatorname{covers} x\} \quad(x \in \mathcal{L}) .
$$

A modular lattice $\mathcal{L}$ is said to be uniform if the ascending operator $(\cdot)^{+}$is defined (i.e., the right hand side of (3.1) exists for all $x$ ) and is an automorphism on $\mathcal{L}$.

Example 3.1. As in $\{0,1\}^{n}$ (see Example 2.3), $\mathbf{Z}^{n}$ becomes a lattice with respect to vector order $\leq$, where $x \wedge y=\min (x, y)$ and $x \vee y=\max (x, y)$. The component sum $x \mapsto \sum_{i=1}^{n} x_{i}$ is a valuation, and hence $\mathbf{Z}^{n}$ is a modular lattice (by Lemma 2.1). The ascending operator is equal to $x \mapsto x+\mathbf{1}$, which is clearly an automorphism. Thus $\mathbf{Z}^{n}$ is a uniform modular lattice.

Example 3.2. Let $T=(V, E)$ be an infinite tree with no vertex of degree one. Regard $T$ as a bipartite graph $\left(V_{0}, V_{1} ; E\right)$, where $V_{0}$ and $V_{1}$ denote two color classes. Define $\ell: V \rightarrow\{0,1\}$ by $\ell(x):=0$ if $x \in V_{0}$ and $\ell(x):=1$ if $x \in V_{1}$. Consider the directed graph on vertex set $V \times \mathbf{Z}$ such that an edge of head $(x, k)$ and tail $\left(x^{\prime}, k^{\prime}\right)$ is given if and only if $x$ and $x^{\prime}$ are adjacent in $T$ and $\ell\left(x^{\prime}\right)+2 k^{\prime}=\ell(x)+2 k+1\left(\Leftrightarrow k=k^{\prime}\right.$ and $\ell\left(x^{\prime}\right)=\ell(x)+1$ or $k^{\prime}=k+1$ and $\left.\ell(x)=\ell\left(x^{\prime}\right)+1\right)$. This graph is acyclic, and naturally induces a partial order on $V \times \mathbf{Z}$. Let $\mathcal{L}$ denote the resulting poset. For an infinite path $P$ in $T$, the subposet $V(P) \times \mathbf{Z}$ for vertex set of $V(P)$ of $P$ is isomorphic to $\mathbf{Z}^{2}$. The join and meet of two points $x, y$ exist in $\mathbf{Z}^{2}=V(P) \times \mathbf{Z}$ for an infinite path $P$ containing $x, y$. One can see that the function $(x, k) \mapsto \ell(x)+2 k$ is a valuation on $\mathcal{L}$. Hence $\mathcal{L}$ is a modular lattice. Since every vertex has at least two neighbors, the ascending operator coincides with $(x, k) \mapsto(x, k+1)$, which is clearly an automorphism on $\mathcal{L}$. Thus $\mathcal{L}$ is a uniform modular lattice.

Example 3.3 (See also [1, Section 5.15]). Let $K$ be a field with a discrete valuation $v$, that is, a function $v: K \rightarrow \mathbf{Z} \cup\{\infty\}$ satisfying $v(x y)=v(x)+v(y), v(x)+v(y) \geq$ $\min (v(x), v(y))$, and $v(x)=0 \Leftrightarrow x=0$. Let $R:=\{x \in K \mid v(x) \geq 0\}$ be the associated valuation ring. It is known that $R$ is a PID and has a unique maximal ideal $m=\{x \in K \mid v(x)>0\}$. The ideal $m$ is generated by an element $t \in K$ (called a uniformizer). Consider the $K$-vector space $K^{n}$, which is also regarded as an $R$-module. Let $\mathcal{L}$ be the family of all free $R$-submodules of $K^{n}$ with rank $n$. Such a module (called a lattice in the literature [8, Section 19]) is precisely an $R$-submodule generated by a 
$K$-linear basis $v_{1}, v_{2}, \ldots, v_{n}$ of $K^{n}$. Regard $\mathcal{L}$ as a poset with respect to the inclusion order. Then $\mathcal{L}$ is a uniform modular lattice, where the ascending operator is given by $\mathcal{L} \ni L \mapsto t^{-1} L$. To see this fact, first note a general fact that the family of all submodules of any module becomes a modular lattice with $\wedge=\cap$ and $\vee=+$; indeed it is easy to see $L \cap(N+M)=L \cap N+M$ for $M \subseteq L$. Therefore, to see that $\mathcal{L}$ is a modular lattice, it suffices to verify that $L, M \in \mathcal{L}$ implies $L \cap M, L+M \in \mathcal{L}$. This is immediate from $L \cap M \subseteq L \subseteq L+M \subseteq t^{-k}(L \cap M) \subseteq t^{-k} L$ for large $k \in \mathbf{Z}$ and the fact that every $R$-submodule of a free $R$-module (with PID $R$ ) is free. Define $h: \mathcal{L} \rightarrow \mathbf{Z}$ by

$$
h(L):=v\left(\operatorname{det}\left(v_{1} v_{2} \cdots v_{n}\right)\right) \quad(L \in \mathcal{L})
$$

where $L$ is generated by a basis $v_{1}, v_{2}, \ldots, v_{n}$ of $K^{n}$. Then $h(L)$ is independent of the choice of the basis. Observe that for $L, M \in \mathcal{L}$ with $L \subseteq M$ it holds $h(L) \geq h(M)$ and holds $h(L)>h(M)$ if and only if $L \neq M$. (In fact, $-h$ is a valuation of $\mathcal{L}$ in the sense of Section 2.2.) From this (and discreteness of $v$ ), we see that $\mathcal{L}$ satisfies $(\mathrm{F})$. Also, if $L$ is covered by $M$, then $M=L+R t^{-1} v$ for some $v \in L$. From this, we see that the ascending operator of $\mathcal{L}$ is given by $L \mapsto t^{-1} L$ and is obviously an automorphism.

A particular example of such a field $K$ is the field $F(t)$ of rational functions over a field $F$. The valuation $v$ is given by $v(p / q):=\operatorname{deg} p-\operatorname{deg} q$ with two polynomials $p, q$, where deg takes the minimum degree of a polynomial.

Above examples actually provide representatives of affine buildings of type A. In Section 3.1, we show that any uniform modular lattice $\mathcal{L}$ yields an affine building of type A. In Section 3.2, we show the reverse construction.

\subsection{Uniform modular lattices $\Rightarrow$ affine buildings of type $A$}

Let $\mathcal{L}$ be a uniform modular lattice. Let $(\cdot)^{-}: \mathcal{L} \rightarrow \mathcal{L}$ denote the inverse of the ascending operator $(\cdot)^{+}$.

Lemma 3.4. The inverse $(\cdot)^{-}$of $(\cdot)^{+}$is given by

$$
(x)^{-}=\bigwedge\{w \in \mathcal{L} \mid w \text { is covered by } x\} \quad(x \in \mathcal{L}) .
$$

In particular, the opposite $\breve{\mathcal{L}}$ of $\mathcal{L}$ is a uniform modular lattice.

Proof. By definition, $(x)^{+}$is the join of all atoms of $\left[x,(x)^{+}\right]$. Hence $\left[x,(x)^{+}\right]$is a complemented modular lattice (by $(\mathrm{cm} 1)$ ). We show that if $y \in \mathcal{L}$ is covered by $(x)^{+}$, then $y$ belongs to $\left[x,(x)^{+}\right]$, i.e., $x \preceq y$. Indeed, since $(\cdot)^{+}$is an automorphism, there is $y^{\prime} \in \mathcal{L}$ such that $\left(y^{\prime}\right)^{+}=y$. Also $x$ covers $y^{\prime}$, which implies $x \preceq\left(y^{\prime}\right)^{+}$by the definition of $(\cdot)^{+}$. The opposite of $\left[x,(x)^{+}\right]$is also complemented modular (by $(\mathrm{cm} 3)$ ). Therefore $x$ is the meet of all elements (coatoms) covered by $(x)^{+}$in $\left[x,(x)^{+}\right]$. By the above argument, they are exactly elements covered by $(x)^{+}$in $\mathcal{L}$. This means that the right hand side of $(3.2)$ exists, and equal to $(x)^{-}$.

For an integer $k \in \mathbf{Z}$, let $(\cdot)^{+k}$ be defined as $\left((\cdot)^{+(k-1)}\right)^{+}$if $k>0,\left((\cdot)^{+(k+1)}\right)^{-}$if $k<0$, and the identity map if $k=0$. For $k>0$, we also denote $(\cdot)^{+(-k)}$ by $(\cdot)^{-k}$.

Lemma 3.5. For $x, y \in \mathcal{L}$, the intervals $\left[x,(x)^{+}\right]$and $\left[y,(y)^{+}\right]$are complemented modular lattices of the same rank. 
Proof. We show that $\left[x,(x)^{+}\right]$and $\left[y,(y)^{+}\right]$have the same rank. It suffices to consider the case where $y$ covers $x$ (by $(\mathrm{F}))$. Since $(\cdot)^{+}$is an automorphism, $(y)^{+}$covers $(x)^{+}$. Therefore we have $1+r\left[y,(y)^{+}\right]=r\left[x,(y)^{+}\right]=r\left[x,(x)^{+}\right]+1$ (by (JD)), which implies $r\left[x,(x)^{+}\right]=r\left[y,(y)^{+}\right]$.

The uniform-rank of $\mathcal{L}$ is defined as the rank $r\left[x,(x)^{+}\right]$of interval $\left[x,(x)^{+}\right]$for $x \in \mathcal{L}$. A chain $x^{0} \prec x^{1} \prec \cdots \prec x^{m}$ is said to be short if $x^{m} \preceq\left(x^{0}\right)^{+}$. Define an equivalence relation $\sim$ on $\mathcal{L}$ by $x \sim y$ if $(x)^{+k}=y$ for some $k \in \mathbf{Z}$. Let $\mathcal{C}(\mathcal{L})$ be the simplicial complex on $\mathcal{L} / \sim$ consisting of all short chains in $\mathcal{L}$ modulo $\sim$. The goal of this section is to show the following.

Theorem 3.6. Let $\mathcal{L}$ be a uniform modular lattice of uniform-rank $n \geq 2$. Then the simplicial complex $\mathcal{C}(\mathcal{L})$ is an affine building of type $A$ with dimension $n-1$.

Let us return to the above Examples 3.1, 3.2, and 3.3. For $\mathcal{L}=\mathbf{Z}^{n}$ (Example 3.1), the simplicial complex $\mathcal{C}(\mathcal{L})$ is nothing but the affine Coxeter complex of type A, since any maximal short chain is the form of (2.5) and the ascending operator is $x \mapsto x+\mathbf{1}$. In the case of Example $3.2, \mathcal{C}(\mathcal{L})$ is regarded as the original tree $T$. It is well-known that an infinite tree without vertices of degree one is a 1-dimensional affine building (of type A). In Example 3.3, the complex $\mathcal{C}(\mathcal{L})$ is nothing but the affine building for $\operatorname{SL}\left(K^{n}\right)$. This is a canonical example of an affine building of type A; see [8, Section 19]. Apartments are given by $\mathcal{C}(\mathcal{L}(Q))$ for the sublattice $\mathcal{L}(Q)$ of $\mathcal{L}$ consisting of modules $R t^{\alpha_{1}} v_{1}+R t^{\alpha_{2}} v_{2}+\cdots R t^{\alpha_{n}} v_{n}$ for nonsingular $Q=\left(v_{1} v_{2} \cdots v_{n}\right) \in K^{n \times n}$ and $\alpha \in \mathbf{Z}^{n}$. Observe that $\mathcal{L}(Q)$ is isomorphic to the opposite of $\mathbf{Z}^{n}$ with $(x)^{+}=x-\mathbf{1}$ for $x \in$ $\mathcal{L}(Q)=\mathbf{Z}^{n}$, and $\mathcal{C}(\mathcal{L}(Q))$ is isomorphic to the affine Coxeter complex of type A. The definition of uniform modular lattice is inspired by this example.

In the following, we suppose that the uniform-rank of $\mathcal{L}$ is equal to $n$. Motivated by the above $\mathcal{L}(Q)$, define a $\mathbf{Z}^{n}$-skeleton of $\mathcal{L}$ by a sublattice $\mathcal{F}$ that is isomorphic to $\mathbf{Z}^{n}$ and satisfies $(x)^{+}=x+\mathbf{1}$ for all $x \in \mathcal{F}$, where $x \mapsto x+\mathbf{1}$ is the ascending operator in $\mathcal{F}=\mathbf{Z}^{n}$. The proof of Theorem 3.6 goes along precisely the same line of the proof of Theorem 2.8. Thus we show the following two lemmas. The first one corresponds to Lemma 2.6, and will be proved later.

Lemma 3.7. For two short chains $C, D$ in $\mathcal{L}$, there exists a $\mathbf{Z}^{n}$-skeleton of $\mathcal{L}$ containing $C, D$.

Let $C=\left(x=x^{0} \prec x^{1} \prec \cdots \prec x^{n}=(x)^{+}\right)$be a maximal short chain. Let us define the relative position $y_{C} \in \mathbf{Z}^{n}$ of an element $y \in \mathcal{L}$ with respect to $C$. Choose a $\mathbf{Z}^{n_{-}}$ skeleton $\mathcal{F}$ containing $C$ and $y$ via Lemma 3.7. Identify $\mathcal{F}$ with $\mathbf{Z}^{n}$ so that $x^{i}-x^{i-1}=e_{i}$ for $i=1,2, \ldots, n$. Define the relative position $y_{C} \in \mathbf{Z}^{n}$ as the integer vector $y-x$ in this coordinate.

Lemma 3.8. The relative position $y_{C}$ of $y \in \mathcal{L}$ is independent of the choice of a $\mathbf{Z}^{n}$ skeleton containing $C, y$.

Proof. We may assume that $x \preceq y$, since $\left((x)^{-}\right)_{C}=x_{C}-\mathbf{1}$ in any $\mathbf{Z}^{n}$-skeleton containing $x$. Define sequence $x=z^{0}, z^{1}, \ldots, z^{k}=y$ in $\mathcal{F}=\mathbf{Z}^{n}$ with $k:=\max _{i} y_{i}-x_{i}$ by

$$
z^{j}:=\left(z^{j-1}+\mathbf{1}\right) \wedge y=z^{j-1}+\sum\left\{e_{i} \mid i: z_{i}^{j-1}<y_{i}\right\} .
$$


Since $z^{j}$ is obtained from $z^{j-1}, y$ by taking the ascending operator and $\wedge$, any $\mathbf{Z}^{n_{-}}$ skeleton containing $z^{j-1}, y$ also contains $z^{j}$. Consequently every $\mathbf{Z}^{n}$-skeleton containing $x, y$ contains the whole sequence $z^{j}$. Now $y_{C}=\sum_{j=1}^{k} \sum\left\{e_{i} \mid i: z_{i}^{j-1}<y_{i}\right\}$. An index $i$ with $z_{i}^{j-1}<y_{i}$ is precisely an index with $\left(x^{i}+(j-1) \mathbf{1}\right) \wedge y \succ\left(x^{i-1}+(j-1) \mathbf{1}\right) \wedge y$. This means that the indices of the sum in (3.3) are independent of the choice of a $\mathbf{Z}^{n}$-skeleton. Thus the relative position $y_{C}$ is independent of the choice of a $\mathbf{Z}^{n}$-skeleton.

Assuming the two lemmas, we complete the proof of Theorem 3.6 .

Proof of Theorem 3.6. Short chains in a $\mathbf{Z}^{n}$-skeleton are short chains in $\mathcal{L}\left(\right.$ by $(x)^{+}=$ $x+1$ ). Therefore $\mathbf{Z}^{n}$-skeletons induce subcomplexes in $\mathcal{C}(\mathcal{L})$. We show that these subcomplexes satisfy the axiom of apartments. Observe that they are isomorphic to the affine Coxeter complex of type A, which implies (B1). Consider two simplices $A, B$ in $\mathcal{C}(\mathcal{L})$, which come from two short chains $C, D$ in $\mathcal{L}$. By Lemma 3.7 there is a $\mathbf{Z}^{n}$-skeleton containing $C, D$. This implies (B2). Suppose that two $\mathbf{Z}^{n}$-skeletons $\mathcal{F}, \mathcal{G}$ contain two short chains $C, D$. Both $\mathcal{F}$ and $\mathcal{G}$ are regarded as $\mathbf{Z}^{n}$. To distinguish them, the unit vectors of $\mathcal{F}$ and of $\mathcal{G}$ are denoted by $e_{1}, e_{2}, \ldots, e_{n}$ and $e_{1}^{\prime}, e_{2}^{\prime}, \ldots, e_{n}^{\prime}$, respectively. By appropriate renumbering and translation, we can assume that $C$ is equal to $\left(\mathbf{0} \leq e_{1} \leq e_{1}+e_{2} \leq \cdots \leq e_{1}+e_{2}+\cdots+e_{n}=\mathbf{1}\right)$ in $\mathcal{F}$ and $\left(\mathbf{0} \leq e_{1}^{\prime} \leq e_{1}^{\prime}+e_{2}^{\prime} \leq\right.$ $\left.\cdots \leq e_{1}^{\prime}+e_{2}^{\prime}+\cdots+e_{n}^{\prime}=\mathbf{1}\right)$ in $\mathcal{G}$. Consider an isomorphism $\varphi: \mathcal{F} \rightarrow \mathcal{G}$ defined by $\sum_{i=1}^{n} z_{i} e_{i} \mapsto \sum_{i=1}^{n} z_{i} e_{i}^{\prime}$. The map $\varphi$ obviously induces a bijection between short chains. Moreover, by Lemma 3.8, $\varphi$ is the identity on the set $\mathcal{F} \cap \mathcal{G}$ of all common points. In particular, $\varphi$ is the identity on $C \cup D$. Hence $\varphi$ induces an isomorphism with (B3).

The remainder of this section is devoted to proving Lemma 3.7. In the following, the rank $r[x, y]$ of interval $[x, y]$ is denoted by $r_{x}(y)$. The function $y \mapsto r_{x}(y)$ is the rank function of the sublattice consisting of elements $y$ with $y \succeq x$.

We start with studying representations of $\mathbf{Z}^{n}$-skeletons. A segment is a chain $a^{0} \prec$ $a^{1} \prec \cdots \prec a^{s}$ such that $a^{l}$ covers $a^{l-1}$ for $l=1,2, \ldots, s$, and $a^{l+1} \notin\left[a^{l-1},\left(a^{l-1}\right)^{+}\right]\left(\ni a^{l}\right)$ for $l=1,2, \ldots, s-1$. A ray is an infinite chain $a^{0} \prec a^{1} \prec \cdots \prec a^{l} \prec \cdots$ satisfying this property for all $l=1,2, \ldots$ If $x=a^{0}$, a segment and a ray are called an $x$-segment and $x$-ray, respectively.

Lemma 3.9. A segment in $\mathcal{L}$ is a segment in the opposite $\breve{\mathcal{L}}$.

Proof. $a^{l+1} \notin\left[a^{l-1},\left(a^{l-1}\right)^{+}\right]$implies $a^{l+1} \wedge\left(a^{l-1}\right)^{+}=a^{l}$. Then $\left(a^{l+1}\right)^{-} \wedge a^{l-1}=\left(a^{l}\right)^{-} \prec$ $\left(a^{l+1}\right)^{-}$. This implies that $a^{l-1} \nsucceq\left(a^{l+1}\right)^{-}$, and $a^{l-1} \notin\left[\left(a^{l+1}\right)^{-}, a^{l+1}\right]$. Hence $\left(a^{l}\right)$ is a segment in $\check{\mathcal{L}}$.

Lemma 3.10. Any x-segment can be extended to an x-ray.

Proof. Let $x=a^{0} \prec a^{1} \prec \cdots \prec a^{s}$ be an $x$-segment. Since $\left(a^{s-1}\right)^{+}$is covered by $\left(a^{s}\right)^{+}$, and $\left[a^{s},\left(a^{s}\right)^{+}\right]$is complemented (by Lemma 3.5), we can choose an atom $a^{s+1}$ in $\left[a^{s},\left(a^{s}\right)^{+}\right]$such that $a^{s+1} \vee\left(a^{s-1}\right)^{+}=\left(a^{s}\right)^{+}$; in particular $a^{s+1} \notin\left[a^{s-1},\left(a^{s-1}\right)^{+}\right]$. Then $a^{0} \prec a^{1} \prec \cdots \prec a^{s} \prec a^{s+1}$ is a segment. Repeating this process, we obtain a ray $a^{0} \prec a^{1} \prec \cdots \prec a^{s} \prec \cdots$.

$x$-segments (or $x$-rays) $\left(x=a_{i}^{0} \prec a_{i}^{1} \prec a_{i}^{2} \prec \cdots\right)(i=1,2, \ldots, k)$ are said to be independent if $a_{1}^{1}, a_{2}^{1}, \ldots, a_{k}^{1}$ are independent atoms in $\left[x,(x)^{+}\right]$, i.e., $r_{x}\left(a_{1}^{1} \vee a_{2}^{1} \vee \cdots \vee a_{k}^{1}\right)=$ $k$. An ordered set $\alpha=\left(a_{i}^{l}\right)_{i, l}$ of $k$ independent $x$-segments is called a partial $k$-frame at $x$. Let $\langle\alpha\rangle$ denote the sublattice generated by all $a_{i, l}$ in $\alpha$. 
Lemma 3.11. Let $\alpha=\left(a_{i}^{l}\right)_{i=1,2, \ldots, k, l=0,1, \ldots, s_{i}}$ be a partial $k$-frame at $x$. For an element $p \in \mathcal{L}$ satisfying $p \wedge\left(\bigvee_{i} a_{i}^{1}\right)=x$, define $b_{i}^{l}$ by

$$
b_{i}^{l}:=p \vee a_{i}^{l} \quad\left(i=1,2, \ldots, k, l=0,1,2, \ldots, s_{i}\right) .
$$

Then $\beta:=\left(b_{i}^{l}\right)_{i, l}$ is a partial $k$-frame at $p$, where the map $u \mapsto p \vee u$ is an isomorphism from $\langle\alpha\rangle$ to $\langle\beta\rangle$ with $r[x, p]=r[u, p \vee u]$.

Proof. It suffices to prove the statement for the case where $p$ covers $x$. We first show, by induction on $l$, that $b_{i}^{l}$ covers $a_{i}^{l}$ and $b_{i}^{l-1}$ for any $i=1,2, \ldots, k$. Here we let $b^{l}:=b_{i}^{l}$ and $a^{l}:=a_{i}^{l}$ for simplicity. In the case of $l=1$, this is true by $p \wedge\left(\bigvee_{i} a_{i}^{1}\right)=x=a^{0}$ (and equality (2.1) for $r_{x}$ ). Suppose that $l>1$ and that $b^{l-1}$ covers $a^{l-1}$ and $b^{l-2}$. Necessarily $p=b^{0} \npreceq a^{l}$ for $l^{\prime} \leq l-1$. If $p \preceq a^{l}$, then it must hold $a^{l}=b^{l}=b^{l-1}=a^{l-1} \vee b^{l-2} \in$ $\left[a^{l-2},\left(a^{l-2}\right)^{+}\right]$; this is a contradiction to $a^{l} \notin\left[a^{l-2},\left(a^{l-2}\right)^{+}\right]$. Thus $b^{l}=p \vee a^{l}$ covers both of $a^{l}$ and $b^{l-1}$.

Next we show that $b^{l+1} \notin\left[b^{l-1},\left(b^{l-1}\right)^{+}\right]$. Now $b^{l}$ covers $a^{l}$ and $b^{l-1}$. Necessarily $\left(b^{l}\right)^{+}$ covers $\left(a^{l}\right)^{+}$and $\left(b^{l-1}\right)^{+}$; in particular $\left(b^{l}\right)^{+}=\left(a^{l}\right)^{+} \vee\left(b^{l-1}\right)^{+}$. Also $a^{l+1} \vee\left(a^{l-1}\right)^{+}=\left(a^{l}\right)^{+}$ must hold (since $\left.\left[a^{l-1},\left(a^{l-1}\right)^{+}\right] \not \supset a^{l+1} \in\left[a^{l},\left(a^{l}\right)^{+}\right]\right)$. By $a^{l+1} \preceq b^{l+1}=b^{l} \vee a^{l+1} \preceq\left(a^{l}\right)^{+}$, we have $\left(a^{l}\right)^{+}=a^{l+1} \vee\left(a^{l-1}\right)^{+} \preceq b^{l+1} \vee\left(a^{l-1}\right)^{+} \preceq\left(a^{l}\right)^{+}$, implying $b^{l+1} \vee\left(a^{l-1}\right)^{+}=\left(a^{l}\right)^{+}$. Therefore $\left(b^{l}\right)^{+}=\left(a^{l}\right)^{+} \vee\left(b^{l-1}\right)^{+}=b^{l+1} \vee\left(a^{l-1}\right)^{+} \vee\left(b^{l-1}\right)^{+}=b^{l+1} \vee\left(b^{l-1}\right)^{+}$. Since $\left(b^{l}\right)^{+}$ covers $\left(b^{l-1}\right)^{+}$, we have $b^{l+1} \npreceq\left(b^{l-1}\right)^{+}$, as required.

Thus $\left(b_{i}^{l}\right)$ for each $i$ is a $p$-segment. The independence of $b_{1}^{1}, b_{2}^{1}, \ldots, b_{k}^{1}$ follows from $r_{p}\left(b_{1}^{1} \vee b_{2}^{1} \vee \cdots \vee b_{k}^{1}\right)=r_{x}\left(a_{1}^{1} \vee a_{2}^{1} \vee \cdots \vee a_{k}^{1} \vee p\right)-1=r_{x}(p)+r_{x}\left(a_{1}^{1} \vee a_{2}^{1} \vee \cdots \vee a_{k}^{1}\right)-r_{x}(x)-1=k$.

If $u=a_{1}^{z_{1}} \vee a_{2}^{z_{2}} \vee \cdots \vee a_{k}^{z_{k}} \in\langle\alpha\rangle$, then $p \vee u=b_{1}^{z_{1}} \vee b_{2}^{z_{2}} \vee \cdots \vee b_{k}^{z_{k}} \in\langle\beta\rangle$, and the statement for $u \mapsto p \vee u$ is an immediate consequence of the next lemma (and (2.1)).

Let $\alpha=\left(a_{i}^{l}\right)_{i=1,2, \ldots, k, l=0,1, \ldots, s_{i}}$ at a partial $k$-frame at $x$. For an integer vector $z \in$ $[\mathbf{0}, s] \subseteq \mathbf{Z}^{k}$ with $s=\left(s_{1}, s_{2}, \ldots, s_{k}\right)$, define an element $\alpha(z) \in\langle\alpha\rangle$ by

$$
\alpha(z):=a_{1}^{z_{1}} \vee a_{2}^{z_{2}} \vee \cdots \vee a_{k}^{z_{k}} .
$$

Lemma 3.12. For a partial $k$-frame $\alpha=\left(a_{i}^{l}\right)_{i=1,2, \ldots, k, l=0,1, \ldots, s_{i}}$ at $x$, the sublattice $\langle\alpha\rangle$ is isomorphic to $[\mathbf{0}, s] \subseteq \mathbf{Z}^{n}$, where the map $z \mapsto \alpha(z)$ is an isomorphism from $[\mathbf{0}, s]$ to $\langle\alpha\rangle$ such that

$$
r_{x}(\alpha(z))=z_{1}+z_{2}+\cdots+z_{k} .
$$

Proof. We first show equation (3.6) by induction on $k$. In the case of $k=1$, this is obvious. Suppose $k>1$. Then $a_{k}^{z_{k}} \wedge\left(\bigvee_{1 \leq i \leq k-1} a_{i}^{1}\right)=x$ holds. Indeed, by Lemma 2.7 with $p=\bigvee_{1 \leq i \leq k-1} a_{i}^{1}$, chain $\left(p \vee a_{k}^{l}\right)_{l=0,1,2, \ldots, z_{k}}$ is a $p$-segment (of length $\left.z_{k}\right)$. Thus $r\left[x, a_{k}^{z_{k}}\right]=z_{k}=r\left[p, p \vee a_{k}^{z_{k}}\right]=r\left[p \wedge a_{k}^{z_{k}}, a_{k}^{z_{k}}\right]$ and (rc) imply $a_{k}^{z_{k}} \wedge p=x$. Define a partial $(k-1)$-frame $\beta=\left(b_{i}^{l}\right)_{i, l}$ at $a_{k}^{z_{k}}$ according to (3.4) with $p=a_{k}^{z_{k}}$. Then $\alpha(z)=\beta\left(z^{\prime}\right)$ and also $r_{x}(\alpha(z))=z_{k}+r_{p}\left(z^{\prime}\right)$, where $z^{\prime}$ denotes the vector in $\mathbf{Z}^{k-1}$ obtained from $z \in \mathbf{Z}^{k}$ by omitting the $k$-th coordinate $z_{k}$ of $z$. Since $\beta$ is a partial $(k-1)$-frame, by induction we have $r_{p}\left(z^{\prime}\right)=z_{1}+z_{2}+\cdots+z_{k-1}$, from which we obtain (3.6).

Next we show

$$
\left(a_{1}^{z_{1}} \vee a_{2}^{z_{2}} \vee \cdots \vee a_{k-1}^{z_{k-1}}\right) \wedge a_{k}^{z_{k}}=x
$$

Then $(\succeq)$ is obvious. Consider $r_{x}\left(a_{1}^{z_{1}} \vee a_{2}^{z_{2}} \vee \cdots \vee a_{k-1}^{z_{k-1}}\right)+r_{x}\left(a_{k}^{z_{k}}\right)=r_{x}\left(\left(a_{1}^{z_{1}} \vee a_{2}^{z_{2}} \vee \cdots \vee\right.\right.$ $\left.\left.a_{k-1}^{z_{k-1}}\right) \wedge a_{k}^{z_{k}}\right)+r_{x}(\alpha(z))$. By (3.6) and (rc), we have $(=)$.

We are ready to prove the statement. By using (3.7), every element $u$ in $\langle\alpha\rangle$ can be written as $u=\alpha(z)$ for some $z \in[\mathbf{0}, s]$. It suffices to show that this expression is unique. 


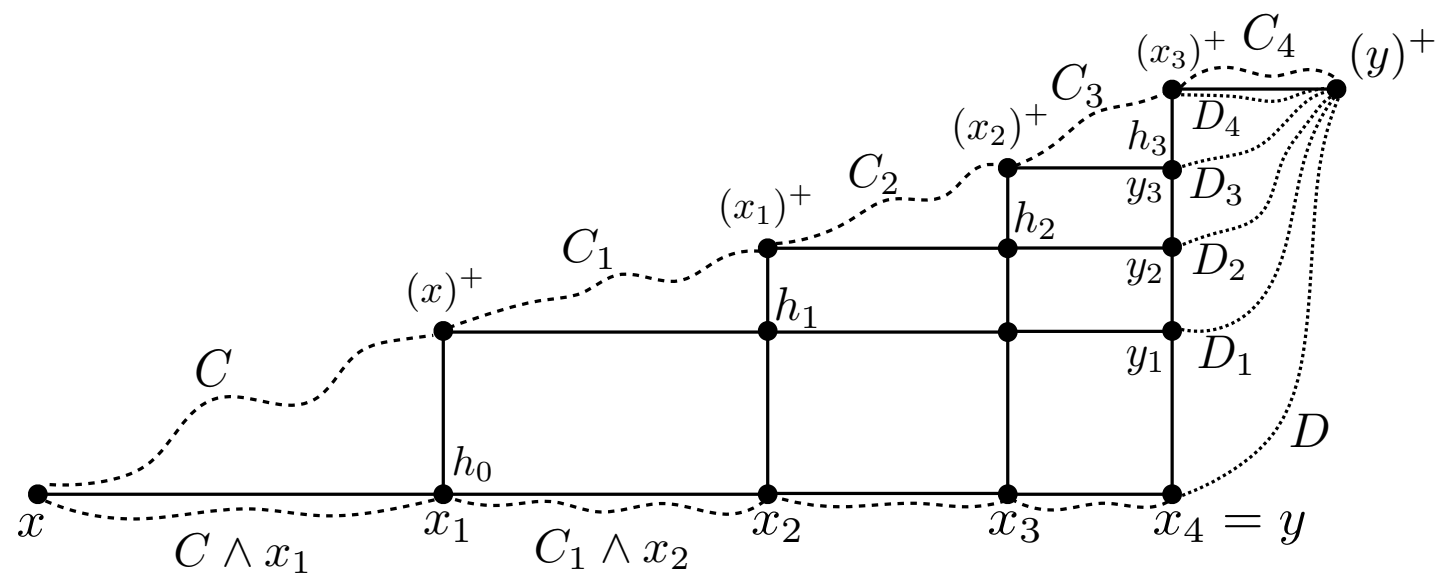

Figure 1: $x_{j}, y_{j}, h_{j}, C_{j}, D_{j}$

For each $i=1,2, \ldots, k$, choose the maximum index $z_{i}^{\prime} \in \mathbf{Z}_{+}$such that $a_{i}^{z_{i}^{\prime}} \preceq u$. Then $z_{i} \leq z_{i}^{\prime}$ (since $a_{i}^{z_{i}} \preceq u$ ). Consider $u^{\prime}:=\alpha\left(z^{\prime}\right)$. Then $u^{\prime} \preceq u$, implying $r_{x}\left(z^{\prime}\right) \leq r_{x}(z)$. On the other hand, $r_{x}(u)=z_{1}+z_{2}+\cdots+z_{k} \leq z_{1}^{\prime}+z_{2}^{\prime}+\cdots+z_{k}^{\prime}=r_{x}\left(u^{\prime}\right)$. Thus, by (rc), it must hold $u=u^{\prime}$ and $z_{i}=z_{i}^{\prime}$ for $i=1,2, \ldots, k$.

An ordered set of $n$ independent rays (at $x$ ) is particularly called a frame. For a frame $\alpha=\left(a_{i}^{l}\right)$, the sublattice $\langle\alpha\rangle$ is isomorphic to $\mathbf{Z}_{+}^{n}:=(\mathbf{Z} \cap[0, \infty))^{n}$ so that $(\alpha(z))^{+}=\alpha(z+\mathbf{1})$.

Lemma 3.13. The sublattice of $\mathcal{L}$ is a $\mathbf{Z}^{n}$-skeleton if and only if it is equal to $\bigcup_{k=0,1,2, \ldots}\langle\alpha\rangle^{-k}$ for a frame $\alpha$.

Proof. Let $\mathcal{F}$ be a $\mathbf{Z}^{n}$-skeleton, and identified with $\mathbf{Z}^{n}$. Observe that the chain $\left(l e_{i}\right)_{l=0,1,2, \ldots}$ is a $\mathbf{0}$-ray. Then the set $\alpha=\left(l e_{i}\right)_{i, l}$ is a frame with $\mathcal{F}=\mathbf{Z}^{n}=\bigcup_{k=0,1,2, \ldots}\left(\mathbf{Z}_{+}^{n}-k \mathbf{1}\right)=$ $\bigcup_{k=0,1,2, \ldots}\langle\alpha\rangle^{-k}$. Let $\alpha=\left(a_{i}^{l}\right)_{i, l}$ be a frame. It is easy to see that $\langle\alpha\rangle^{-k}$ is isomorphic to $(\mathbf{Z} \cap[-k, \infty))^{n}$ by $z \mapsto(\alpha(z+k \mathbf{1}))^{-k}$. Also it holds $\langle\alpha\rangle^{-k} \subseteq\langle\alpha\rangle^{-k-1}$ since $\alpha(z)^{-k}=\alpha(z+\mathbf{1})^{-k-1}$. Therefore $\bigcup_{k=0,1,2, \ldots}\langle\alpha\rangle^{-k}$ is a $\mathbf{Z}^{n}$-skeleton.

Lemma 3.14. For $x, y \in \mathcal{L}$, there is $k \geq 0$ such that $x \preceq(y)^{+k}$.

Proof. We may assume that $x \npreceq y$. Hence $x \succ x \wedge y$. Choose an atom $a$ in $[x \wedge y, x]$. By $a \wedge y=x \wedge y$ and modularity equality (2.1) for $r_{x \wedge y}, a \vee y$ is an atom in $\left[y,(y)^{+}\right]$. Consequently $x \wedge y \prec a \preceq x \wedge(y)^{+}$. Thus, for $k \geq r[x \wedge y, x]$, it holds $x \wedge(y)^{+k}=x$, implying $x \preceq(y)^{+k}$.

Proof of Lemma 3.7. We may assume that both $C$ and $D$ are maximal short chains (of length $n)$. Suppose that $C \subseteq\left[x,(x)^{+}\right]$and $D \subseteq\left[y,(y)^{+}\right]$. We may assume that $x \preceq y$. Indeed, for the general case, choose $k$ such that $x \preceq(y)^{+k}$ by Lemma 3.14. Then $y^{\prime}:=(y)^{+k}$ satisfies $x \preceq y^{\prime}$. Any $\mathbf{Z}^{n}$-skeleton containing $C$ and $(D)^{+k} \subseteq\left[y^{\prime},\left(y^{\prime}\right)^{+}\right]$, also contains $C$ and $D$.

We first define elements $x_{j}, y_{j}, h_{j}$ and chains $C_{j}, D_{j}$, along an intuition in Figure 1 . For $j=0,1,2, \ldots$, define $x_{j}$ by $x_{0}:=x$ and

$$
\begin{aligned}
x_{j} & :=\left(x_{j-1}\right)^{+} \wedge y \\
& =\bigvee\left\{a \mid a \text { is } x_{j-1} \text { or an atom in }\left[x_{j-1}, y\right]\right\} \quad \in\left[x_{j-1},\left(x_{j-1}\right)^{+}\right],
\end{aligned}
$$


where the (second) equality follows from the observation that $\left(x_{j-1}\right)^{+} \wedge y$ belongs to complemented modular lattice $\left[x_{j-1},\left(x_{j-1}\right)^{+}\right]$(by $x_{j-1} \preceq y$ ) and hence $\left(x_{j-1}\right)^{+} \wedge y$ is the join of atoms $a$ in $\left[x_{j-1},\left(x_{j-1}\right)^{+}\right]$(with $a \preceq y$ ). For some $m(\leq r[x, y]$ ), it holds $x=x_{0} \prec x_{1} \prec \cdots \prec x_{m}=x_{m+1}=\cdots=y$. For $j=0,1,2, \ldots, m$, define short chain $C_{j}$ by $C_{0}:=C$ and

$$
C_{j}:=\left(C_{j-1} \wedge x_{j}\right)^{+} .
$$

Since $C_{j-1} \wedge x_{j}$ is a chain in $\left[x_{j-1}, x_{j}\right], C_{j}$ is a chain in $\left[\left(x_{j-1}\right)^{+},\left(x_{j}\right)^{+}\right]$. Define $y_{j}$ and $D_{j}$ by $y_{0}=y$ and $D_{0}=D$, and

$$
\begin{aligned}
y_{j} & :=\left(x_{j-1}\right)^{+} \vee y, \\
D_{j} & :=D_{j-1} \vee y_{j}=D \vee y_{j} .
\end{aligned}
$$

Finally define $h_{j}$ by $h_{0}:=x_{1}$ and

$$
h_{j}:=x_{j+1} \vee\left(x_{j-1}\right)^{+}=\left(x_{j}\right)^{+} \wedge y_{j}
$$

where the last equality follows from $x_{j+1} \vee\left(x_{j-1}\right)^{+}=\left(\left(x_{j}\right)^{+} \wedge y\right) \vee\left(x_{j-1}\right)^{+}=\left(x_{j}\right)^{+} \wedge$ $\left(y \vee\left(x_{j-1}\right)^{+}\right)=\left(x_{j}\right)^{+} \wedge y_{j}$.

Now $C_{m}$ and $D_{m}$ are maximal chains in complemented modular lattice $\left[\left(x_{m-1}\right)^{+},(y)^{+}\right]$. By Lemma 2.6, there is a basis $a_{1}, a_{2}, \ldots, a_{k}$ of $\left[\left(x_{m-1}\right)^{+},(y)^{+}\right]$such that $\left\langle a_{1}, a_{2}, \ldots, a_{k}\right\rangle$ contains $C_{m}$ and $D_{m}$. In particular, $\alpha:=\left(\left(x_{m-1}\right)^{+}, a_{i}\right)_{i=1,2, \ldots k}$ is a partial $k$-frame at $\left(x_{m-1}\right)^{+}$such that $\langle\alpha\rangle$ contains $C_{m}$ and $D_{m}$.

We are going to show that a (given) partial $k$-frame $\alpha=\left(a_{i}^{l}\right)_{i=1, \ldots, k, l=0,1, \ldots, s_{i}}$, at $\left(x_{j}\right)^{+}$ with $\langle\alpha\rangle \supseteq C_{j+1}, D_{j+1}$ can be extended to a partial $k^{\prime}$-frame $\beta=\left(b_{i}^{l}\right)_{i, l}$ at $\left(x_{j-1}\right)^{+}$with $\langle\beta\rangle \supseteq C_{j}, D_{j}$, where we let $x_{-1}:=(x)^{-}$so that $\left(x_{-1}\right)^{+}=x$. The case of $j=0$ is our goal. Figure 2 illustrates an intuition behind the argument we proceed with. We assume that $\alpha(\mathbf{1})=\left(x_{j+1}\right)^{+}$and $\alpha(s)=(y)^{+}$for $s:=\left(s_{1}, s_{2}, \ldots, s_{k}\right)$; this is true for the base case of the induction. Let $b_{i}^{l}$ be defined by

$$
b_{i}^{l}:=\left(x_{j-1}\right)^{+} \vee\left(a_{i}^{l}\right)^{-} \quad\left(i=1,2, \ldots, k, l=0,1,2, \ldots, s_{i}\right) .
$$

Obviously $\left(\left(a_{i}^{l}\right)^{-}\right)$is a partial $k$-frame at $x_{j}$. By $\left(x_{j-1}\right)^{+} \wedge(\alpha(\mathbf{1}))^{-}=\left(x_{j-1}\right)^{+} \wedge x_{j+1}=x_{j}$ and Lemma 3.11, $\beta:=\left(b_{i}^{l}\right)_{i, l}$ is a partial $k$-frame at $\left(x_{j-1}\right)^{+}$. Here $\langle\beta\rangle$ contains $C_{j} \wedge h_{j}$ since $\langle\beta\rangle \supseteq\left(x_{j-1}\right)^{+} \vee\left(C_{j+1}\right)^{-}=\left(x_{j-1}\right)^{+} \vee\left(C_{j} \wedge x_{j+1}\right)=C_{j} \wedge\left(x_{j+1} \vee\left(x_{j-1}\right)^{+}\right)=C_{j} \wedge h_{j}$; the last equality follows from 3.8$)$. Also $\beta(s)=\left(x_{j-1}\right)^{+} \vee(\alpha(s))^{-}=\left(x_{j-1}\right)^{+} \vee\left((y)^{+}\right)^{-}=$ $\left(x_{j-1}\right)^{+} \vee y=y_{j}$. Choose any complement $u$ of $y_{j+1}$ in $\left[y_{j},(y)^{+}\right] \subseteq\left[y,(y)^{+}\right]$such that $\left\langle\left[y_{j}, y_{j+1}\right] \cup\left[y_{j}, u\right]\right\rangle$ contains $D_{j}$ (via $(\mathrm{cm} 2)$ and Lemma 2.5 (1)). Then $u$ is also a complement of $\left(x_{j}\right)^{+}$in $\left[h_{j},(y)^{+}\right]$. Indeed, $y_{j+1}=\left(x_{j}\right)^{+} \vee y=\left(x_{j}\right)^{+} \vee y_{j}$ implies that $\left(x_{j}\right)^{+} \vee u=(y)^{+}$. By this fact with (2.1) for $r_{h_{j}}, r\left[u,(y)^{+}\right]=r\left[y_{j}, y_{j+1}\right]=r\left[h_{j},\left(x_{j}\right)^{+}\right]$ (by (3.8)) and the rank-comparison argument (rc) for $h_{j} \preceq\left(x_{j}\right)^{+} \wedge u$, we conclude $\left(x_{j}\right)^{+} \wedge u=h_{j}$.

Claim. For $z \in[\mathbf{0}, s]$, the following hold:

(1) $\beta\left(z+e_{i}\right) \preceq \alpha(z) \wedge u$ if $z_{i}<s_{i}$.

(2) $r[\beta(z), \alpha(z) \wedge u]=k$.

In particular, $\beta(z+\mathbf{1})=\alpha(z) \wedge u$ for $z \in[\mathbf{0}, s-\mathbf{1}]$. 


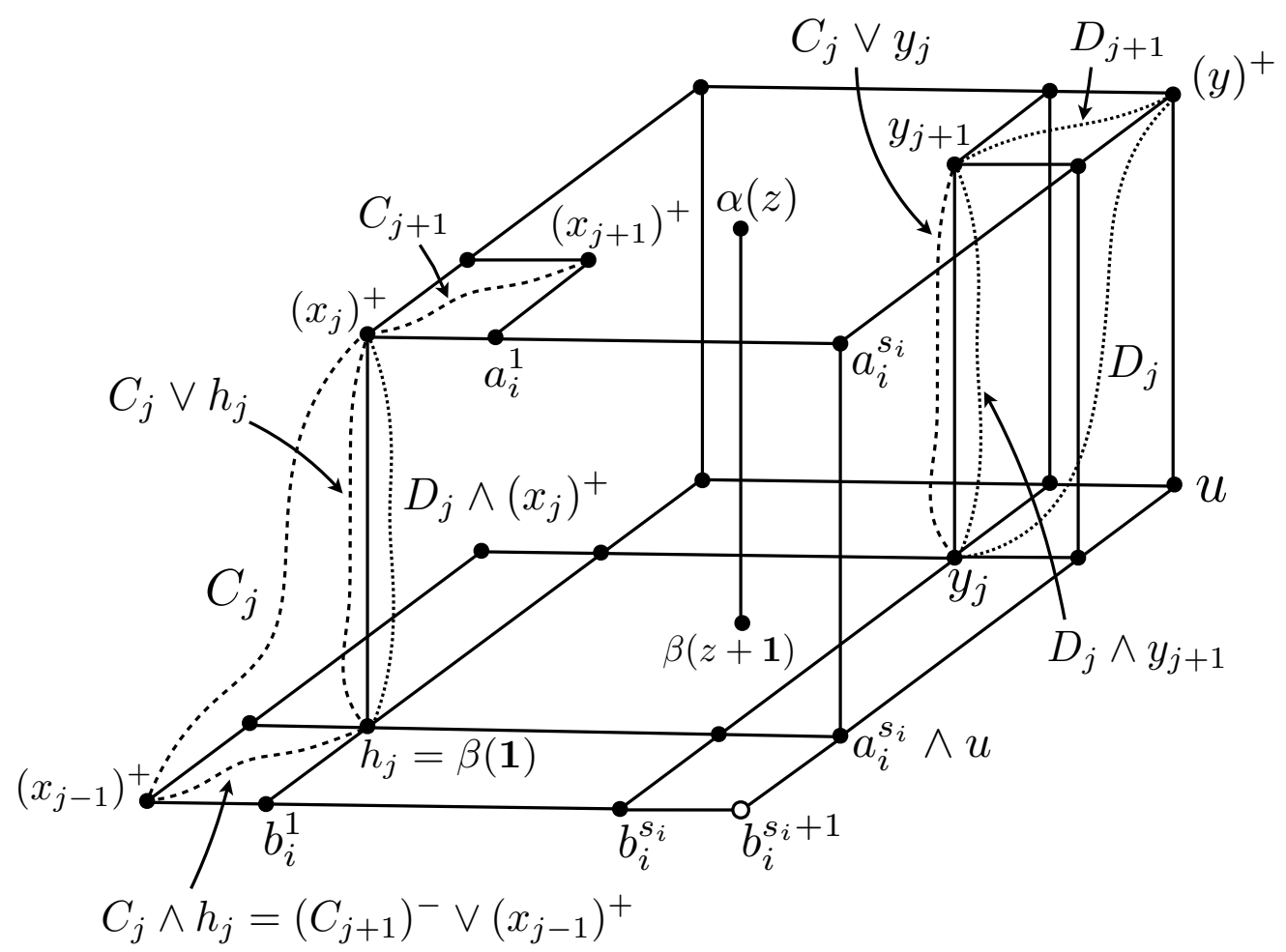

Figure 2: Extending a partial $k$-frame

Proof. We first show $\beta(z) \preceq \alpha(z) \wedge u$. Obviously $\beta(z) \preceq \beta(s)=y_{j} \prec u$. We show $\beta(z) \preceq \alpha(z)$. Since $\left(x_{j-1}\right)^{+}$is the join of atoms in $\left[x_{j},\left(x_{j}\right)^{+}\right]$and $(\alpha(z))^{-}$ is a complement of $\left(x_{j-1}\right)^{+}$in $\left[x_{j}, \beta(z)\right]$ (by Lemmas 3.11 and 3.12), $\beta(z)$ is also the join of atoms in $\left[\alpha(z)^{-}, \alpha(z)\right]$ (by Lemma $2.2(1)$ ), implying $\beta(z) \preceq \alpha(z)$ with $r\left[x_{j},\left(x_{j-1}\right)^{+}\right]=r\left[(\alpha(z))^{-}, \beta(z)\right]$.

Here $u$ is a complement of $\left(x_{j}\right)^{+}$in $\left[h_{j},(y)^{+}\right]$. This implies that $r\left[h_{j},\left(x_{j}\right)^{+}\right]=$ $r[\alpha(z) \wedge u, \alpha(z)]\left(\right.$ via Lemma 2.2). Now $n=r\left[x_{j},\left(x_{j}\right)^{+}\right]=r\left[x_{j},\left(x_{j-1}\right)^{+}\right]+r\left[\left(x_{j-1}\right)^{+}, h_{j}\right]+$ $r\left[h_{j},\left(x_{j}\right)^{+}\right]$and $n=r\left[(\alpha(z))^{-}, \alpha(z)\right]=r\left[(\alpha(z))^{-}, \beta(z)\right]+r[\beta(z), \alpha(z) \wedge u]+r[\alpha(z) \wedge$ $u, \alpha(z)]$ (by (JD) and Lemma 3.5). Hence we have (2) $k=r\left[\left(x_{j-1}\right)^{+}, h_{j}\right]=r[\beta(z), \alpha(z) \wedge$ $u$. Suppose $z_{i}<s_{i}$. Then $\beta\left(z+e_{i}\right):=\left(x_{i-1}\right)^{+} \vee\left(\alpha\left(z+e_{i}\right)\right)^{-}=\beta(z) \vee\left(\alpha\left(z+e_{i}\right)\right)^{-}$. Here $\beta(z)$ is the join of atoms in $\left[(\alpha(z))^{-}, \alpha(z)\right]$ (as seen above), and $\left(\alpha\left(z+e_{i}\right)\right)^{-}$is an atom of $\left[\alpha(z)^{-}, \alpha(z)\right]$. This means that $\beta\left(z+e_{i}\right) \preceq \alpha(z)$. Thus we have (1). Now $\beta(z+\mathbf{1})=\alpha(z) \wedge u$ for $z \in[\mathbf{0}, s-\mathbf{1}]$ follows from the combination of (2), (rc) for $\beta(z+\mathbf{1}) \preceq \alpha(z) \wedge u$ (by (1)), and Lemma 3.12 .

By this claim, for each $i=1,2, \ldots, k, \beta\left(\mathbf{1}+\left(s_{i}-1\right) e_{i}\right)$ is covered by $a_{i}^{s_{i}} \wedge u=$ $\alpha\left(s_{i} e_{i}\right) \wedge u$. Since $\left[b_{i}^{s_{i}}, a_{i}^{s_{i}}\right] \subseteq\left[\left(a_{i}^{s_{i}}\right)^{-}, a_{i}^{s_{i}}\right]$ is complemented modular, we can choose a complement $b_{i}^{s_{i}+1}$ of $\beta\left(\mathbf{1}+\left(s_{i}-1\right) e_{i}\right)$ in $\left[b_{i}^{s_{i}}, a_{i}^{s_{i}} \wedge u\right]$, which covers $b_{i}^{s_{i}}$; see Figure 2 .

We show that $\left(b_{i}^{l}\right)_{l=0,1, \ldots, s_{i}+1}$ is a segment. By Lemmas 3.9 and 3.11 for the opposite $\check{\mathcal{L}}$, we have that $\left(a_{i}^{s_{i}-2}, a_{i}^{s_{i}-1}, a_{i}^{s_{i}}\right) \wedge u$ is a segment. By the above claim, we must have $\left(a_{i}^{s_{i}-2}, a_{i}^{s_{i}-1}, a_{i}^{s_{i}}\right) \wedge u=\left(\beta\left(\mathbf{1}+\left(s_{i}-2\right) s_{i}\right), \beta\left(\mathbf{1}+\left(s_{i}-1\right) s_{i}\right), a_{i}^{s_{i}} \wedge u\right)$. Similarly, $\left.\left(b_{i}^{s_{i}-1}, b_{i}^{s_{i}}, b_{i}^{s_{i}+1}\right)=\left(\left(\beta\left(\mathbf{1}+\left(s_{i}-2\right) e_{i}\right), \beta\left(\mathbf{1}+\left(s_{i}-1\right) e_{i}\right), \alpha\left(s_{i} e_{i}\right)\right) \wedge u\right)\right) \wedge b_{i}^{s_{i}+1}$ is a segment. This concludes that $\left(b_{i}^{l}\right)_{l=0,1, \ldots, s_{i}+1}$ is a segment.

Now $\beta=\left(b_{i}^{l}\right)_{i=1,2, \ldots, k, l=0,1, \ldots, s_{i}+1}$ is a partial $k$-frame at $\left(x_{j-1}\right)^{+}$such that $\beta(\mathbf{1})=h_{j}$ 
and $\beta(s+\mathbf{1})=u$. By Lemma 3.11, the above claim, $b_{i}^{s_{i}+1} \preceq u$, and (rc), we must have $\beta(z+\mathbf{1})=\alpha(z) \wedge u$ for all $z \in[\mathbf{0}, s]$, which in turn implies $\beta(z+\mathbf{1}) \vee\left(x_{j}\right)^{+}=\alpha(z)$ (by Lemma 2.2 (1)). Consider two short chains $C_{j} \vee y_{j}$ and $D_{j} \wedge y_{j}$ in $\left[y_{j}, y_{j+1}\right]$. Choose a basis $c_{1}, c_{2}, \ldots, c_{i^{\prime}}$ of $\left[y_{j}, y_{j+1}\right]$ that generates $C_{j} \vee y_{j}$ and $D_{j} \wedge y_{j+1}$. By Lemma 2.5, the sublattice $\left\langle c_{1}, c_{2}, \ldots, c_{i^{\prime}}, \beta\left(s+e_{1}\right), \beta\left(s+e_{2}\right), \ldots, \beta\left(s+e_{k}\right)\right\rangle$ contains $D_{j}$. Also $c_{1} \wedge\left(x_{j}\right)^{+}, c_{2} \wedge\left(x_{j}\right)^{+}, \ldots, c_{i^{\prime}} \wedge\left(x_{j}\right)^{+}$is a basis of $\left[h_{j},\left(x_{j}\right)^{+}\right]$(by Lemma 2.2 (1)) that generates $C_{j} \vee h_{j}$ and $D_{j} \wedge\left(x_{j}\right)^{+}$. Choose a complement $v$ of $h_{j}$ in $\left[\left(x_{j-1}\right)^{+},\left(x_{j}\right)^{+}\right]$such that $\left\langle\left[\left(x_{j-1}\right)^{+}, v\right] \cup\left[\left(x_{j-1}\right)^{+}, h_{j}\right]\right\rangle$ contains $C_{j}$ (via Lemma 2.5). Here $c_{1} \wedge v, c_{2} \wedge v, \ldots, c_{i^{\prime}} \wedge v$ is a basis of $\left[\left(x_{j-1}\right)^{+}, v\right]$ (by Lemma $\left.2.2(1)\right)$. Append segment $\left(\left(x_{j-1}\right)^{+}, c_{i} \wedge v\right.$ ) to $\beta$ for $i=1,2, \ldots, i^{\prime}$. Then we obtain a partial $\left(k+i^{\prime}\right)$-frame $\beta$ at $\left(x_{j-1}\right)^{+}$such that $\langle\beta\rangle$ contains $C_{j}$ and $D_{j}$ (since $\left.\left(c_{i} \wedge v\right) \vee \beta(s)=c_{i}\right)$, and $\beta(\mathbf{1})=\left(x_{j}\right)^{+}$and $\beta(s+\mathbf{1})=(y)^{+}$, as required.

For $j=0$, we obtain a partial $n$-frame $\alpha$ at $x$ that generates $C$ and $D$. Extend each $x$-segment in $\alpha$ to an $x$-ray according to Lemma 3.12. Thus we obtain a frame $\alpha$ that generates $C$ and $D$, and obtain a $\mathbf{Z}^{n}$-skeleton $\bigcup_{k=0,1,2, \ldots}\langle\alpha\rangle^{-k}$ containing $C$ and $D$ (Lemma 3.13). This completes the proof of Lemma 3.7.

\subsection{Affine buildings of type $\mathrm{A} \Rightarrow$ uniform modular lattices}

Let $\Delta$ be an affine building of type A with dimension $n-1$. We first introduce a special labeling analogous to a natural coloring in the spherical case. Consider the subposet $\Lambda$ of $\mathbf{Z}^{n}$ defined by

$$
\Lambda:=\left\{\begin{array}{l|l}
x \in \mathbf{Z}^{n} & 0 \leq \sum_{i=1}^{n} x_{i} \leq n-1
\end{array}\right\} .
$$

For every point $x \in \mathbf{Z}^{n}$, there is a unique $x^{\prime} \in \Lambda$ with $x-x^{\prime} \in \mathbf{Z} \mathbf{1}$. Namely $\Lambda$ is the set of representatives of $\mathbf{Z}^{n} / \mathbf{R} \mathbf{1}$. Consider the order complex $\mathcal{O}(\Lambda)$ of $\Lambda$. Then one can observe that the affine Coxeter complex of type $\mathrm{A}$ is isomorphic to the subcomplex $\mathcal{O}^{\prime}(\Lambda)$ of $\mathcal{O}(\Lambda)$ consisting of chains $x_{0}<x_{1}<\cdots<x_{m}$ with $x_{m}<x_{0}+\mathbf{1}$.

A coloring $\ell: \Delta^{0} \rightarrow\{0,1,2, \ldots, n-1\}$ of $\Delta$ is said to be natural if for every apartment $\Sigma$ there is an isomorphism $\varrho: \Sigma \rightarrow \mathcal{O}^{\prime}(\Lambda)$ with $\ell(x)=\sum_{i=1}^{n} \varrho(x)_{i}$ for all $x \in \Sigma^{0}$.

Lemma 3.15. A natural coloring exists.

From the group-theoretic view, a natural coloring corresponds to the ordering of generators of the affine Coxeter group of type A so that consecutive numbers are assigned to adjacent generators in the Dynkin diagram of type $\tilde{A}_{n}$ (that is a cycle).

Proof. Before constructing a natural coloring, we note one remark on automorphisms on $\mathcal{O}(\Lambda)$. For $z \in \mathbf{Z}^{n}$, define $\kappa_{z}^{ \pm}: \Lambda \rightarrow \Lambda$ so that $\kappa_{z}^{ \pm}(x)$ is the unique point in $\Lambda$ with $\pm x+z-\kappa_{z}^{ \pm}(x) \in \mathbf{R} 1$. Then $\kappa_{z}^{ \pm}$induces an automorphism on $\mathcal{O}^{\prime}(\Lambda)$ with $\sum_{i} \kappa_{z}^{ \pm}(x)_{i}=\sum_{i}\left( \pm x_{i}+z_{i}\right) \bmod n$.

Let us start the proof of this lemma. Consider an arbitrary apartment $\Sigma$. Choose an arbitrary isomorphism $\varrho: \Sigma \rightarrow \mathcal{O}^{\prime}(\Lambda)$. Define the color $\ell(x)$ of $x \in \Sigma^{0}$ by $\ell(x):=$ $\sum_{i=1}^{n} \varrho(x)_{i} \in\{0,1, \ldots, n-1\}$. This is a coloring of $\Sigma$. Extend this coloring to the whole coloring $\ell: \Delta^{0} \rightarrow\{0,1, \ldots, n-1\}$; see (col) in Section 2.3 . We verify that $\ell$ is indeed natural. Here we observe:

(*) In $\Sigma$, every vertex $x$ is adjacent to $\left(\begin{array}{c}n \\ |k-\ell(x)|\end{array}\right)$ vertices of color $k \neq \ell(x)$. 
Consider another apartment $\Sigma^{\prime}$. Suppose first that $\Sigma$ and $\Sigma^{\prime}$ have a common maximal simplex $A$. Choose an isomorphism $\Sigma \rightarrow \Sigma^{\prime}$ fixing $A$ via (B3). This isomorphism is taken to be color-preserving by $\left(\mathrm{B}^{+}\right)$. Therefore the property $(*)$ holds in $\Sigma^{\prime}$. Consider an isomorphism $\varrho^{\prime}: \Sigma^{\prime} \rightarrow \mathcal{O}^{\prime}(\Lambda)$. Now $\ell^{\prime}: \Sigma^{\prime 0} \rightarrow\{0,1, \ldots, n-1\}$ defined by $x \mapsto \sum_{i=1}^{n} \varrho^{\prime}(x)_{i}$ is a coloring on $\Sigma^{\prime}$. By replacing $\ell^{\prime}$ by $\kappa_{v}^{ \pm} \circ \ell^{\prime}$ if necessarily, we can assume that $\ell^{\prime}(x)=0$ for the vertex $x \in A$ with $\ell(x)=0$ and $\ell^{\prime}(y) \neq n-1$ for the vertex $y \in A$ with $\ell(y)=1$. In $\Sigma^{\prime}$, each vertex $x$ must satisfy $(*)$ for $\ell^{\prime}$. By the uniqueness of coloring on $\Sigma^{\prime}$, there is a bijection $\sigma$ on $\{0,1, \ldots, n-1\}$ such that $\ell^{\prime}=\sigma \circ \ell$. Then it must hold $\sigma(0)=0$ and $\sigma(1) \neq n-1$. All vertices in $A$ satisfy $(*)$ for $\ell$ and for $\ell^{\prime}$. Therefore $\left(\begin{array}{c}n \\ |k-l|\end{array}\right)=\left(\begin{array}{c}n \\ |\sigma(k)-\sigma(l)|\end{array}\right)$ must hold for $k \neq l$. For $|k-l|=1$ or $n-1$, it holds $n=\left(\begin{array}{c}n \\ |\sigma(k)-\sigma(l)|\end{array}\right)$, which implies $|\sigma(k)-\sigma(l)|=1$ or $n-1$. By $\sigma(0)=0$ and $\sigma(1) \neq n-1$, it holds $\sigma(1)=1$, consequently, $\sigma(2)=2, \sigma(3)=3, \ldots$. Thus $\sigma$ is the identity, and $\varrho^{\prime}$ is a desired isomorphism.

Next suppose that $\Sigma^{\prime}$ is arbitrary. By (B3), there is an apartment $\Sigma^{\prime \prime}$ containing a maximal simplex in $\Sigma$ and a maximal simplex $B$ in $\Sigma^{\prime \prime}$. Apply the above argument with replacing $\Sigma^{\prime}$ by $\Sigma^{\prime \prime}$ and $A$ by $B$. Then we obtain a desired isomorphism from $\Sigma^{\prime}$ to $\mathcal{O}^{\prime}(\Lambda)$.

Fix a natural coloring $\ell$. We construct a uniform modular lattice from $\Delta$. Our construction generalizes that in Example 3.2. Consider a directed graph $G(\Delta)$ on vertex set $\Delta^{0} \times \mathbf{Z}$, where two distinct vertices $(x, k)$ and $\left(x^{\prime}, k^{\prime}\right)$ have an edge from $\left(x^{\prime}, k^{\prime}\right)$ to $(x, k)$, denoted by $\left(x^{\prime}, k^{\prime}\right) \rightarrow(x, k)$, if $x$ and $x^{\prime}$ belong to a common simplex in $\Delta$ and $\ell\left(x^{\prime}\right)+k^{\prime} n=\ell(x)+k n+1\left(\Leftrightarrow\right.$ either $k^{\prime}=k$ and $\ell\left(x^{\prime}\right)=\ell(x)+1$ or $k^{\prime}=k+1$ and $\left.\ell(x)=\ell\left(x^{\prime}\right)+n-1\right)$. The graph $G(\Delta)$ is acyclic, since $(x, k) \mapsto \ell(x)+n k$ is monotone decreasing on any directed path. Define a partial order $\preceq$ on $\Delta^{0} \times \mathbf{Z}$ by $(x, k) \preceq\left(x^{\prime}, k^{\prime}\right)$ if there is a directed path on $G(\Delta)$ from $\left(x^{\prime}, k^{\prime}\right)$ to $(x, k)$. The resulting poset on $\Delta^{0} \times \mathbf{Z}$ satisfies $(\mathrm{F})$, and is denoted by $\mathcal{L}(\Delta)$.

Theorem 3.16. Let $\Delta$ be an affine building of type $A$. Then $\mathcal{L}(\Delta)$ is a uniform modular lattice, where $\Delta$ is equal to $\mathcal{C}(\mathcal{L}(\Delta))$.

The rest of this section is devoted to proving this theorem. Now each apartment $\Sigma$ can be regarded as $\mathcal{O}^{\prime}(\Lambda)$, where $\ell(x)=\sum_{i=1}^{n} x_{i}$ for $x \in \Sigma^{0}=\Lambda$. Let $\mathcal{L}(\Sigma)$ denote the subposet of $\mathcal{L}(\Delta)$ consisting of $(x, k) \in \Sigma^{0} \times \mathbf{Z}$. Consider the canonical retraction $\rho_{\Sigma}=\rho_{\Sigma, A}: \Delta \rightarrow \Sigma$ (for some $A \in \Sigma$ ); see (ret) in Section 2.3. From this, we define an order-preserving retraction $\bar{\rho}_{\Sigma}: \mathcal{L}(\Delta) \rightarrow \mathcal{L}(\Sigma)$ by $(x, k) \mapsto\left(\rho_{\Sigma}(x), k\right)$. Under identification $\Sigma^{0}=\Lambda \subseteq \mathbf{Z}^{n}$, we have:

Lemma 3.17. $\mathcal{L}(\Sigma)$ is isomorphic to $\mathbf{Z}^{n}$ by $(x, k) \mapsto x+k \mathbf{1}$.

Proof. From $\mathcal{L}(\Sigma)=\Sigma^{0} \times \mathbf{Z}=\Lambda \times \mathbf{Z}$, we can easily see that the map is a bijection. In particular, we can identify $\mathcal{L}(\Sigma)$ with $\mathbf{Z}^{n}$. We need to show that $p \preceq q$ if and only if $p \leq q$ in $\mathcal{L}(\Sigma)=\mathbf{Z}^{n}$. If $p \leq q$, then there is a directed path from $q$ to $p$ in the Hasse diagram of $\mathbf{Z}^{n}$; it is a directed path in $G(\Delta)$, implying $p \preceq q$. Suppose that $p \preceq q$. There is a directed path $P$ from $q$ to $p$ in $G(\Delta)$. Then the image of $P$ by retraction $\bar{\rho}_{\Sigma}: \mathcal{L}(\Delta) \rightarrow \mathcal{L}(\Sigma)$ is a directed path from $q$ to $p$ in $\mathcal{L}(\Sigma)=\mathbf{Z}^{n}$. This means that $p \leq q$, as required.

As mentioned in Section 2.3, the geometric realization $|\Sigma|$ of an apartment $\Sigma$ is naturally regarded as $\mathbf{R}^{n} / \mathbf{R} \mathbf{1}$ with $\Sigma^{0}=\mathbf{Z}^{n} / \mathbf{R} \mathbf{1}$. The next lemma is crucial for showing the existence of the meet and join in $\mathcal{L}(\Delta)$. 
Lemma 3.18. For $x, y \in \mathbf{Z}^{n}=\mathcal{L}(\Sigma)$, let $\left(\bar{x}^{k}\right)_{k}$ and $\left(\underline{x}^{k}\right)_{k}$ be sequences of points in $\mathbf{Z}^{n}$ defined by

$$
\begin{aligned}
\bar{x}^{k} & :=\bar{x}^{k-1}+\sum\left\{e_{i} \mid i: y_{i}>\bar{x}_{i}^{k-1}\right\} \\
\underline{x}^{k} & :=\underline{x}^{k-1}-\sum\left\{e_{i} \mid i: y_{i}<\underline{x}_{i}^{k-1}\right\} \quad(k=1,2, \ldots),
\end{aligned}
$$

where $\bar{x}^{0}=\underline{x}^{0}:=x$, and $\bar{x}^{k}=\max (x, y)$ and $\underline{x}^{k}=\min (x, y)$ for large $k$. Then the sequences $\left(\bar{x}^{k}+\mathbf{R} \mathbf{1}\right)_{k}$ and $\left(\underline{x}^{k}+\mathbf{R} \mathbf{1}\right)_{k}$ of vertices in $\Sigma^{0}$ belong to every apartment containing $x+\mathbf{R} \mathbf{1}$ and $y+\mathbf{R} \mathbf{1}$.

Proof. We first show a property of the triangulation $\Sigma$ of $\mathbf{R}^{n} / \mathbf{R} \mathbf{1}$.

Claim. For a vector $u \in \mathbf{R}^{n}$, the simplex of $|\Sigma|$ containing $u+\mathbf{R} \mathbf{1}$ in its relative interior contains $\lceil u\rceil+\mathbf{R} \mathbf{1}$ and $\lfloor u\rfloor+\mathbf{R} \mathbf{1}$ as vertices.

Here $\lceil u\rceil$ (resp. $\lfloor u\rfloor$ ) is the integral vector obtained from $u$ by rounding up (resp. down) the fractional part of each component of $u$.

Proof. Consider the unique expression $u-\lfloor u\rfloor=\sum_{k=1}^{n} \lambda_{k} \sum_{i \in X_{k}} e_{i}$ for $\emptyset \neq X_{1} \subset X_{2} \subset$ $\cdots \subset X_{n-1} \subset X_{n}=\{1,2, \ldots, n\}$ and $\lambda_{i} \geq 0$ with $\sum_{i=1}^{n} \lambda_{i}<1$. From this, we see that $u$ is a convex combination of $\lfloor u\rfloor$ and $\lfloor u\rfloor+\sum_{i \in X_{k}} e_{i}$ for $k$ with $\lambda_{k}>0$, which forms the simplex in $|\Sigma|$ containing $u$ as its relative interior. Notice that $\lfloor u\rfloor+\sum_{i \in X_{k}} e_{i}$ for largest $k$ with $\lambda_{k}>0$ is equal to $\lceil u\rceil$. This proves the claim.

We prove the lemma. We use the CAT(0)-metrization of $|\Delta|$; see Section 2.3 . The unique geodesic between $\bar{x}^{k}+\mathbf{R} \mathbf{1}$ and $y+\mathbf{R} \mathbf{1}$ in $|\Delta|$ is given by $t \mapsto(1-t) \bar{x}^{k}+t y+\mathbf{R} \mathbf{1} \in$ $\mathbf{R}^{n} / \mathbf{R} \mathbf{1}=|\Sigma|$. By the uniqueness (geo) of the geodesic, every simplex meeting the geodesic in its relative interior must belong to every apartment containing $\bar{x}^{k}+\mathbf{R} \mathbf{1}$ and $y+\mathbf{R} 1$. Notice that for small $\epsilon>0$, the point $\left\lceil\bar{x}^{k}+\epsilon\left(y-\bar{x}^{k}\right)\right\rceil$ is equal to $\bar{x}^{k+1}$. By the claim, $\bar{x}^{k+1}+\mathbf{R} \mathbf{1}$ is a vertex of a simplex with which the geodesic meets in its relative interior. This means that $\bar{x}^{k+1}+\mathbf{R} \mathbf{1}$ belongs to every apartment containing $\bar{x}^{k}+\mathbf{R} \mathbf{1}$ and $y+\mathbf{R} \mathbf{1}$. Consequently, the whole sequence $\left(\bar{x}^{k}+\mathbf{R} \mathbf{1}\right)$ belongs to every apartment containing $x+\mathbf{R} 1$ and $y+\mathbf{R} 1$. The statement for $\underline{x}^{k}$ is shown by replacing $\bar{x}^{k}$ and $\lceil\cdot\rceil$ with $\underline{x}^{k}$ and $\lceil\cdot\urcorner$, respectively.

Proof of Theorem 3.16. We first show that $\mathcal{L}(\Delta)$ is a lattice. Consider any two elements $(x, k)$ and $(y, l)$ of $\mathcal{L}(\Delta)$. By (B2), there is an apartment $\Sigma$ containing $x$ and $y$. By Lemma 3.17, $(x, k)$ and $(y, l)$ are regarded as integer vectors $p=x+k \mathbf{1}$ and $q=y+l \mathbf{1}$, respectively. Thus, in $\mathcal{L}(\Delta)=\mathbf{Z}^{n}$, we can consider the meet $p \wedge_{\Sigma} q:=\min (p, q)$ and the join $p \vee_{\Sigma} q:=\max (p, q)$. We show that $p \wedge_{\Sigma} q$ and $p \vee_{\Sigma} q$ are independent of the choice of the apartment $\Sigma$. Consider another apartment $\Sigma^{\prime}$ containing $p+\mathbf{R} \mathbf{1}$ and $q+\mathbf{R} 1$. By Lemma 3.18, $\Sigma^{\prime}$ contains $p \vee_{\Sigma} q+\mathbf{R} 1$, and hence $\mathcal{L}\left(\Sigma^{\prime}\right)$ contains $p \vee_{\Sigma} q$. Conversely, $\mathcal{L}(\Sigma)$ contains $p \vee_{\Sigma^{\prime}} q$. Consider the order-preserving retraction $\bar{\rho}_{\Sigma}$. We have $p \vee_{\Sigma} q \preceq \bar{\rho}_{\Sigma}\left(p \vee_{\Sigma^{\prime}} q\right)=p \vee_{\Sigma^{\prime}} q$. Also, by considering $\bar{\rho}_{\Sigma^{\prime}}$, we have $p \vee_{\Sigma} q \preceq p \vee_{\Sigma^{\prime}} q$. Thus $p \vee_{\Sigma} q=p \vee_{\Sigma^{\prime}} q$, and operator $\vee:=\vee_{\Sigma}$ is independent of an apartment. Similarly, $\wedge:=\wedge_{\Sigma}$ is well-defined. We show that $p \wedge q$ indeed equals the meet of $p$ and $q$. Consider any common lower bound $u$ of $p$ and $q$. We prove $p \wedge q \succeq u$ by the induction on the (minimum) length $k$ of a directed path $P$ from $p$ to $u$. In the case of $k=0$, we have $u=p=p \wedge q$. Suppose that $k>0$. Consider the next element $p^{\prime}$ following $p$ in $P$. Here $u$ is also a common lower bound of $p^{\prime}$ and $q$. By induction $p^{\prime} \wedge q \succeq u$. Also $p+\mathbf{R} \mathbf{1}$ 
and $p^{\prime}+\mathbf{R} 1$ belong to a common simplex. There is an apartment $\Sigma$ such that $\mathcal{L}(\Sigma)$ contains $p, p^{\prime}$ and $q$. Then $\mathcal{L}(\Sigma)\left(=\mathbf{Z}^{n}\right)$ also contains $p \wedge q$ and $p^{\prime} \wedge q$ with $p \wedge q \succeq p^{\prime} \wedge q$, which implies $p \wedge q \succeq u$, as required. By the same argument, $p \vee q$ is the join of $p$ and $q$.

We show that $\mathcal{L}(\Delta)$ is a modular lattice. Define $v: \mathcal{L}(\Delta) \rightarrow \mathbf{Z}$ by $(x, k) \mapsto \ell(x)+k n$. Then $(x, k) \prec\left(x^{\prime}, k^{\prime}\right)$ implies $v(x, k)<v\left(x^{\prime}, k^{\prime}\right)$. For any apartment $\Sigma$, sublattice $\mathcal{L}(\Sigma)$ is identified with $\mathbf{Z}^{n}$, as shown above. In this identification, any element $(x, k) \in \mathcal{L}(\Sigma)$ is regarded as $p=x+k \mathbf{1} \in \mathbf{Z}^{n}$. By $\ell(x)=\sum_{i} x_{i}$, we have $v(p)=\ell(x)+k n=$ $\sum_{i}(x+k \mathbf{1})_{i}=\sum_{i} p_{i}$. Hence modular equality 2.1$)$ holds on $\mathcal{L}(\Sigma)$ for any apartment $\Sigma$, and holds on the whole $\mathcal{L}(\Delta)$ by $(\mathrm{B} 1)$. Thus $v$ is a valuation on $\mathcal{L}(\Delta)$, and $\mathcal{L}(\Delta)$ is a modular lattice (by Lemma 2.1).

We finally verify that $\mathcal{L}(\Delta)$ is uniform. We show that the ascending operator $(\cdot)^{+}$ coincides with the map $(x, k) \mapsto(x, k+1)$, which is obviously an automorphism on $\mathcal{L}(\Delta)$. If $\left(x^{\prime}, k^{\prime}\right)$ covers $(x, k)$, then $\left(x^{\prime}, k^{\prime}\right) \preceq(x, k+1)$ holds. Therefore it suffices to show that $(x, k+1)$ is the join of some elements that covers $(x, k)$. Consider $\mathcal{L}(\Sigma)$ containing $(x, k)$, which also contains $(x, k+1)$. By Lemma 3.17, $(x, k)$ and $(x, k+1)$ are regarded as integer vectors $x+k \mathbf{1}$ and $x+(k+1) \mathbf{1}$, respectively. Then $x+(k+1) \mathbf{1}$ $(=(x, k+1))$ is the join of $x+k \mathbf{1}+e_{i}$ for $i=1,2, \ldots, n$ (that covers $\left.x+k \mathbf{1}\right)$. Thus the ascending operator equals the map $(x, k) \mapsto(x, k+1)$, and we conclude that $\mathcal{L}(\Delta)$ is a uniform modular lattice with $\Delta=\mathcal{C}(\mathcal{L}(\Delta))$.

\section{Concluding remarks}

We close this paper with a few remarks.

Modular graphs and affine buildings. In [7], we explored interesting connections between CAT(0)-spaces and various subclasses of weakly modular graphs. Among them, orientable modular graphs form a fascinating subclass of weakly modular graphs. They are defined as connected undirected graphs $G=(V, E)$ satisfying:

- For any triple of vertices $x_{1}, x_{2}, x_{3} \in V$ there is a vertex $y \in V$ such that $d\left(x_{i}, x_{j}\right)=$ $d\left(x_{i}, y\right)+d\left(y, x_{j}\right)$ for $1 \leq i<j \leq 3$, where $d$ is the graph metric on $V$.

- There is an edge-orientation such that every 4 -cycle $\left(x_{0}, x_{1}, x_{2}, x_{3}\right)$ is oriented as $x_{i} \rightarrow x_{i+1}$ if and only if $x_{i+2} \leftarrow x_{i+3}$.

(A graph satisfying the first condition is called a modular graph.) It is shown in [7, Section 6.8] that an affine building $\Delta$ of type $C$, which also becomes a CAT(0)-space, gives rise to an orientable modular graph $G$ as a certain subgraph of the 1-skeleton of $\Delta$, in which the graph $G$ recovers original $\Delta$ completely. This raises a natural question: do other affine buildings admit such a graph-theoretic approach by orientable or more generally weakly modular graph? The presented result may be an answer of this question for type A, since the (undirected) Hasse diagram of a modular lattice is an orientable modular graph.

L-convex functions on uniform modular lattices. The primary motivation of uniform modular lattices comes from a recent movement [10, 11, 12] of Discrete Convex Analysis beyond $\mathbf{Z}^{n}$. Originally Discrete Convex Analysis (DCA) [16] was a theory of 
"convex" functions on $\mathbf{Z}^{n}$ generalizing matroids and submodular functions in combinatorial optimization. In DCA, L-convex functions constitute one of fundamental classes of discrete convex functions on $\mathbf{Z}^{n}$. They are defined as functions $g: \mathbf{Z}^{n} \rightarrow \mathbf{R} \cup\{\infty\}$ that satisfy the submodularity inequality

$$
g(x)+g(y) \geq g(\min (x, y))+g(\max (x, y)) \quad\left(x, y \in \mathbf{Z}^{n}\right)
$$

and satisfy the linearity over 1 -direction

$$
g(x+k \mathbf{1})=g(x)+k \alpha \quad\left(x \in \mathbf{Z}^{n}, k \in \mathbf{Z}\right)
$$

for some $\alpha \in \mathbf{R}$. Recent work [10, 11] shows that analogues of L-convex functions are definable on certain grid-like structures generalizing $\mathbf{Z}^{n}$ and bring meaningful applications to several combinatorial optimization problems with which the previous DCA could not deal. In particular, [11] introduces L-convex functions on an affine building of type $C$, and links them to the design of efficient algorithms for classes of network optimization problems; see also [12].

The concept of uniform modular lattices enables us to define what should be called L-convex functions on an affine building of type $A$. Recall Example 3.1 that $\mathbf{Z}^{n}$ is a uniform modular lattice with ascending operator $x \mapsto x+1$. Then the above definition of the L-convexity is naturally extended to an arbitrary uniform modular lattice $\mathcal{L}$. A function $g: \mathcal{L} \rightarrow \mathbf{R} \cup\{\infty\}$ is called $L$-convex if it satisfies the submodularity inequality

$$
g(x)+g(y) \geq g(x \wedge y)+g(x \vee y) \quad(x, y \in \mathcal{L}),
$$

and satisfies the linearity on the ascending operator

$$
g\left((x)^{+k}\right)=g(x)+k \alpha \quad(x \in \mathcal{L}, k \in \mathbf{Z})
$$

for some $\alpha \in \mathbf{R}$. In the case of $\alpha=0$, an L-convex function $g$ is viewed as the vertex set $\mathcal{L} / \sim$ of the affine building $\mathcal{C}(\mathcal{L})$ of type $\mathrm{A}$.

In the subsequent paper [14, we link, via the affine building for $\operatorname{SL}\left(\mathbf{R}(t)^{n}\right)$ (Example 3.3), this new L-convex function to computation of the degree of the determinants of polynomial matrices; it is well-known in the literature that the deg-det computation of polynomial matrices generalizes and abstracts a number of basic combinatorial optimization problems; see [15].

\section{Acknowledgments}

The author thanks Yuni Iwamasa and Koyo Hayashi for careful reading and helpful comments, and thanks the referee for comments. The work was partially supported by JSPS KAKENHI Grant Numbers 25280004, 26330023, 26280004, 17K00029.

\section{References}

[1] H. Abels: The gallery distance of flags. Order 8 (1991) 77-92.

[2] M. Aigner: Combinatorial Theory. Springer, Berlin, 1979. 
[3] P. Abramenko and K. S. Brown: Buildings-Theory and Applications. Springer, New York, 2008.

[4] F. Bruhat, and J. Tits: Groupes réductifs sur un corps local. Institut des Hautes Études Scientifiques. Publications Mathématiques 41, (1972), 5-251.

[5] G. Birkhoff: Lattice Theory. American Mathematical Society, New York, 1940; 3rd edn., American Mathematical Society, Providence, RI, 1967.

[6] M. R. Bridson and A. Haefliger: Metric Spaces of Non-positive Curvature. Springer-Verlag, Berlin, 1999.

[7] J. Chalopin, V. Chepoi, H. Hirai, and D. Osajda. Weakly modular graphs and nonpositive curvature. Memoirs of the AMS, to appear.

[8] P. B. Garrett, Building and Classical Groups. Chapman \& Hall, London, 1997.

[9] G. Grätzer: Lattice Theory: Foundation. Birkhäuser, Basel, 2011.

[10] H. Hirai: Discrete convexity and polynomial solvability in minimum 0-extension problems. Mathematical Programming, Series A 155 (2016), 1-55.

[11] H. Hirai: L-convexity on graph structures. Journal of the Operations Research Society of Japan 61 (2018), 71-109.

[12] H. Hirai: Discrete Convex Functions on Graphs and Their Algorithmic Applications. In: T. Fukunaga and K. Kawarabayashi (eds.) Combinatorial Optimization and Graph Algorithms, Communications of NII Shonan Meetings, Springer Nature, Singapore, (2017), pp. 67-101.

[13] H. Hirai: Uniform semimodular lattices and valuated matroids. Journal of Combinatorial Theory, Series A 165 (2019), 325-359.

[14] H. Hirai: Computing the degree of determinants via discrete convex optimization on Euclidean buildings. SIAM Journal on Applied Geometry and Algebra, to appear.

[15] K. Murota: Matrices and Matroids for Systems Analysis. Springer-Verlag, Berlin, 2000.

[16] K. Murota: Discrete Convex Analysis. SIAM, Philadelphia, 2003.

[17] R. Scharlau: Buildings. In: F. Buekenhout (ed.) Handbook of Incidence Geometry, NorthHolland, Amsterdam, (1995), pp. 477-645.

[18] J. Tits: Buildings of Spherical Type and Finite BN-pairs. Lecture Notes in Mathematics, Vol. 386. Springer-Verlag, Berlin-New York, 1974. 